\title{
L-Carnitine ameliorates congenital myopathy in a tropomyosin 3 de novo mutation transgenic zebrafish
}

\author{
Po-Jui Hsu ${ }^{1,2,3 \dagger}{ }^{\circ}$, Horng-Dar Wang ${ }^{2 \dagger}$, Yung-Che Tseng ${ }^{4}$, Shao-Wei Pan ${ }^{1}$, Bonifasius Putera Sampurna ${ }^{1}$, \\ Yuh-Jyh Jong ${ }^{5,6,7,8^{*}}$ and Chiou-Hwa Yuh ${ }^{1,5,9,10^{*}}$ [D
}

\begin{abstract}
Background: Congenital myopathy (CM) is a group of clinically and genetically heterogeneous muscle disorders, characterized by muscle weakness and hypotonia from birth. Currently, no definite treatment exists for $\mathrm{CM}$. A de novo mutation in Tropomyosin 3-TPM3(E151G) was identified from a boy diagnosed with CM, previously TPM3(E151A) was reported to cause CM. However, the role of TPM3(E151G) in CM is unknown.
\end{abstract}

Methods: Histopathological, swimming behavior, and muscle endurance were monitored in TPM3 wild-type and mutant transgenic fish, modelling CM. Gene expression profiling of muscle of the transgenic fish were studied through RNAseq, and mitochondria respiration was investigated.

Results: While TPM3(WT) and TPM3(E151A) fish show normal appearance, amazingly a few TPM3(E151G) fish display either no tail, a crooked body in both F0 and F1 adults. Using histochemical staining for the muscle biopsy, we found TPM3(E151G) displays congenital fiber type disproportion and TPM3(E151A) resembles nemaline myopathy. TPM3(E151G) transgenic fish dramatically swimming slower than those in TPM3(WT) and TPM3(E151A) fish measured by DanioVision and T-maze, and exhibit weaker muscle endurance by swimming tunnel instrument. Interestingly, L-carnitine treatment on TPM3(E151G) transgenic larvae significantly improves the muscle endurance by restoring the basal respiration and ATP levels in mitochondria. With RNAseq transcriptomic analysis of the expression profiling from the muscle specimens, it surprisingly discloses large downregulation of genes involved in pathways of sodium, potassium, and calcium channels, which can be rescued by L-carnitine treatment, fatty acid metabolism was differentially dysregulated in TPM3(E151G) fish and rescued by L-carnitine treatment.

Conclusions: These results demonstrate that TPM3(E151G) and TPM3(E151A) exhibit different pathogenicity, also have distinct gene regulatory profiles but the ion channels were downregulated in both mutants, and provides a potential mechanism of action of TPM3 pathophysiology. Our results shed a new light in the future development of potential treatment for TPM3-related CM.

Keywords: Congenital myopathy, Zebrafish, Tropomyosin 3 (TPM3), L-Carnitine

*Correspondence: yjjong@gap.kmu.edu.tw; chyuh@nhri.edu.tw

${ }^{\dagger}$ Po-Jui Hsu and Horng-Dar Wang contribute equally to this work

${ }^{1}$ Institute of Molecular and Genomic Medicine, National Health Research Institutes, Zhunan, Miaoli, Taiwan

${ }^{5}$ Department of Biological Science and Technology, National Chiao Tung University, Hsinchu, Taiwan

Full list of author information is available at the end of the article

\section{Background}

Congenital myopathy $(\mathrm{CM})$ is a group of genetic muscle disorders characterized clinically by generalized hypotonia and muscle weakness present at birth and pathologically by the presence of specific morphological features on muscle biopsy, with estimated prevalence about original author(s) and the source, provide a link to the Creative Commons licence, and indicate if changes were made. The images or other third party material in this article are included in the article's Creative Commons licence, unless indicated otherwise in a credit line to the material. If material is not included in the article's Creative Commons licence and your intended use is not permitted by statutory regulation or exceeds the permitted use, you will need to obtain permission directly from the copyright holder. To view a copy of this licence, visit http://creativecommons.org/licenses/by/4.0/. The Creative Commons Public Domain Dedication waiver (http://creativeco mmons.org/publicdomain/zero/1.0/) applies to the data made available in this article, unless otherwise stated in a credit line to the data. 
1:25,000 [1]. According to the main muscle pathological features, the most common types of CM are cores (central core disease), central nuclei (myotubular and centronuclear myopathy), rods (nemaline myopathy), and selective hypotrophy of type 1 fiber (congenital fiber type disproportion, CFTD). In striated muscles, tropomyosin interacts with the troponin complex to regulate actin-myosin interaction. Mutations of tropomyosin 3 (TPM3) cause CM with nemaline myopathy, cap myopathy, and CFTD [2, 3]. TPM3-related CM were reported to exhibit lowered $\mathrm{Ca}^{2+}$-sensitivity and damaged actomyosin cross-bridge cycling in slow fibers [4] and disturb regulatory function via altered actin affinity and tropomodulin binding [5]. To date, over 61 phenotypes and 44 genotypes of CM are reported [6]. Although, mutations in the different genes may cause the same phenotype and different mutations in the same gene can produce multiple different phenotypes. CM share five common key pathophysiological defects in sarcolemmal and intracellular membrane remodeling and excitation-contraction coupling, mitochondrial distribution and function, myofibrillar force generation, atrophy, and autophagy [7]. Currently, no known treatment exists for any type of CM, only supportive therapy. Using the power of the zebrafish model, researchers are able to establish novel insights into pathomechanisms of $\mathrm{CM}$ and provide the necessary groundwork for future therapy development [8].

$\mathrm{L}$-Carnitine is an amino acid that is widely present in human tissues and can be produced from essential amino acids lysine and methionine. It is responsible for sending fatty acids into the mitochondria to burn smoothly and produce energy. L-Carnitine significantly reduced statin-induced myopathy [9] and skeletal muscle atrophy in rats [10]. L-Carnitine improved exercise performance in human patients with mitochondrial myopathy [11]. L-Carnitine may prevent age-associated muscle protein degradation and regulate mitochondrial homeostasis [12]. Although dietary L-tyrosine supplementation was reported to improve bulbar function, activity levels, and exercise tolerance in some nemaline myopathy patients [13]. However, L-tyrosine supplementation was not able to attenuate the skeletal muscle dysfunction in zebrafish and the dominant skeletal muscle $\alpha$-actin nemaline myopathy in mouse models [14].

A seven-year-old boy patient who had been suffering from generalized hypotonia, muscle weakness with myopathic face, high-arched palate, funnel chest, hyporeflexia, poor feeding, and motor delay since early infancy. He presented severe motor delay having head control at 8 months, was able to sit alone at 12 months, walk alone at 20 months, and still cannot run until seven years of age. As his parents refused the invasive clinical examination such as electromyogram or muscle biopsy for him, thus Dr. Yuh-Jyh Jong diagnosed with his serum DNA by using target gene capture/deep sequencing approach [15]. It revealed a novel and de novo mutation of TPM3 at nucleotide 452 from $A$ to $G$, resulting in amino acid 151 changing from glutamic acid (E) to glycine (G). Previously, a TPM3 mutation changing nucleotide 452 from $A$ to $C$ resulting in amino acid 151 changing from glutamic acid (E) to alanine (A) was reported in a CM patient with cap myopathy [3]. To study the precise pathogenicity between TPM3(E151G) and CM, we generated transgenic zebrafish overexpressing the human wild type (WT) and two mutant TPM3 genes in the muscle of zebrafish for functional analysis and potential drug screening. Here, we show that TPM3(E151G) transgenic zebrafish display much severe $C M$ phenotypes than those from TPM3(E151A) and TPM3(WT) transgenic zebrafish. Remarkably, L-carnitine supplementation ameliorates the swimming speed and muscle endurance as well as rescues the downregulation genes involved in sodium, potassium, and calcium channels in TPM3(E151G) transgenic zebrafish by RNAseq. This study provides insights in the pathogenicity of the novel TPM3(E151G) mutation and a drug screening platform to uncover potential therapies for this rare disease.

\section{Materials and methods}

\section{Zebrafish maintenance}

All zebrafish were maintained at the Zebrafish Core Facility in National Health Research Institute (NHRI, Miaoli, Taiwan). Adult fish were maintained under an automated 14:10 h light/dark cycle and a constant temperature of $28{ }^{\circ} \mathrm{C}$. All experiments involving zebrafish were approved by the Institution Animal Care and Use Committee (IACUC) of the NHRI (protocol No. NHRIIACUC-103122-AE). The Taiwan Zebrafish Core Facility at NHRI or TZeNH is a government-funded core facility, and since 2015, it has been accredited by the Association for Assessment and Accreditation of Laboratory Animal Care International (AAALAC) [16].

\section{Transgenic zebrafish lines}

$A B$ wild-type was used in this study. Other transgenic fish lines, including $\operatorname{Tg}(\mathrm{MLC} 2$ :TPM3(WT);myl 7:EGFP), $\quad \operatorname{Tg}($ MLC2:TPM3(E151A); $m y l 7: E G F P), \quad$ and $\operatorname{Tg}(M L C 2: T P M 3(E 151 G) ; m y l 7: E G F P)$ were generated in this study as described previously using Tol2 Gateway cloning Toolkit $[17,18]$. Primers used for construct preparation are listed in Additional file 1: Table S1.

pME-TPM3(E151A) and pME-TPM3(E151G) were created by QuickChange II site-directed mutagenesis (Agilent Technologies, Santa Clara, CA, USA) from 
pME-TPM3(WT). All primers for mutagenesis are listed in Additional file 1: Table S1. The final expression construct pTol2-MLC2:TPM3(E151A):pA/CG2 was generated using Gateway ${ }^{\circledR}$ LR reaction (Invitrogen, Waltham, Massachusetts, USA) using p5E-MLC2, pME-TPM3(E151A), p3E-polyA, and pDestTol2CG2 vectors. The final expression construct pTol2MLC2:TPM3(E151G):pA/CG2 was generated by Gateway ${ }^{\circledR}$ LR reaction (Invitrogen, Waltham, Massachusetts, USA) using p5E-MLC2, pME-TPM3(E151G), p3E-polyA, and pDestTol2CG2 vectors.

Three constructs were created by Tol2-Gateway cloning, and confirmed by real-time reverse transcriptionPCR (qRT-PCR) and sequencing, and microinjected into $\mathrm{AB}$ wildtype zebrafish embryos. Expressions of transgenes were using by heart specific promoter myl7driven green florescence protein (GFP) expression in 48 hours-post-fertilization (hpf) embryos and sequencing confirmed with genomic DNA extracted from adult fin. The mRNA level of TPM3 transgenic zebrafish were examined by qRT-PCR, which confirmed all three TPM3 transgenic zebrafish expressed human TPM3 gene.

\section{Embryos collection}

Embryos were collected once per week with the best results produced with a regular schedule. The night before embryo collection, adult male and female zebrafish were placed into a mating tank with a clapboard after the last feeding. Next morning, the clapboard was removed to start mating. Embryos were collected after 1 hour, and we removed the dead and unfertilized embryos. We transferred healthy embryos to a clean petri dish containing E3 medium ( $5 \mathrm{mM} \mathrm{NaCl}, 0.17 \mathrm{mM} \mathrm{KCl}$, $0.33 \mathrm{mM} \mathrm{CaCl}$, and $0.33 \mathrm{mM} \mathrm{MgSO}{ }_{4}$ ). We maintained the embryos in an incubator at $28^{\circ} \mathrm{C}$.

\section{Microinjection and selection and confirmation of transgenic zebrafish}

We inserted a needle into the Nanoject $\mathrm{II}^{\mathrm{TM}}$ Nanoliter injector (Drummond Scientific, Broomall, PA, USA). For DNA and RNA co-injection, we injected $2.3 \mathrm{~nL}$ containing $0.05 \%$ phenol red into an embryo at the one-cell stage. We ensured the embryos did not develop past the two-cell stage. After injection, embryos were placed in E3 medium and incubated at $28^{\circ} \mathrm{C}$.

Two days after microinjection, we used a fluorescent microscope to screen the hearts of zebrafish embryo for green fluorescence. When the transgenic zebrafish were 3 months old, DNA was extracted by cutting the fins. After amplification of the target fragment by PCR, we entrusted the DNA Sequencing Core Lab at the National Health Research Institutes to sequence and confirm the target sequence.
Fish length and body weight measurement and morphological analysis

Before examination, the fish was anesthesia using 0.0125\% buffered MS-222 solution (ethyl 3-aminobenzoate methane sulfonic acid salt, Sigma-Aldrich, St. Louis, MI, USA). Total length and standard length were measured using Vernier calipers with $0.1 \mathrm{~mm}$ accuracy. Fish body weight was determined to an accuracy of $0.01 \mathrm{~g}$. For morphological analysis, a digital CMOS X-ray detector (Model 2315, Dexela, London, UK), set to $45 \mathrm{kV}$ and $120 \mathrm{~mA}$, with 2.5 seconds of exposure, was used to view vertebrae of the fish [19].

\section{Tracking of larvae fish swimming behavior}

The zebrafish larvae swimming behavior data were tracked and acquired by using DanioVision equipment (Noldus, Wageningen, The Netherlands). Before the indicated times of development, zebrafish larvae were placed individually into a 48-well plate with $1200 \mu \mathrm{L}$ E3 medium overnight. According to DanioVision manual, we used light-induced visual motor response analysis. The swimming behavior test for zebrafish larvae involved the following conditions: the first $30 \mathrm{~min}$ was required for their adaptation, then we used a light switch (20 min: $10 \mathrm{~min}$ in dark and $10 \mathrm{~min}$ in light) for 2 cycles [20, 21]. The water temperature during the experiment was maintained at $28 \pm 0.5^{\circ} \mathrm{C}$.

\section{T-maze behavior test}

A three armed T-maze was used for our experiment. The stem of the maze (length $36 \mathrm{~cm} \times$ width $11 \mathrm{~cm} \times$ height $20 \mathrm{~cm})$ included the start box $(11 \times 11 \times 20 \mathrm{~cm})$ and each arm of the maze $(25 \times 11 \times 20 \mathrm{~cm})$. Blue or red cellophane was attached to the end of the arm. The T-maze was filled with system water and the temperature was maintained at $28 \pm 1{ }^{\circ} \mathrm{C}$.

For the zebrafish adult behavior test, we used T-maze for memory testing and velocity testing. The T-maze behavior test is divided into three phases: pre-training, training, and testing. In the pre-training phase, adult zebrafish were placed in a $3 \mathrm{~L}$ fish tank when they were 3 months old with a white sponge filled with food to induce the fish to find the white sponge twice each day (10:00 and 15:00) for one week. In the training phase, adult zebrafish were moved to the behavioral room and placed into an individual tank. All fish were acclimatized for $1 \mathrm{~h}$ before placement into the T-maze. During each trial, a zebrafish was placed in the start box for $1 \mathrm{~min}$ with the door closed. Then, the door was raised and closed after the fish had left. A stopwatch was used to measure the time required for the fish to find the white sponge filled with food. If the zebrafish did not leave the start box or find the target within $3 \mathrm{~min}$, the experiment 
was terminated. In training phase, each fish was trained once each day for one week. In the testing phase, adult zebrafish were moved to the behavioral room and placed into an individual tank. All fishes acclimatized for $1 \mathrm{~h}$ before placement into the T-maze. During each trial, a zebrafish was placed in the start box for 1 min with the door closed. Then, the door was raised and closed after the fish had left. A stopwatch was used to measure the time required for the fish to find the white sponge. If the zebrafish did not leave the start box or find the target within $3 \mathrm{~min}$, the experiment was terminated. In testing phase, each fish was tested once per day for one week.

\section{Analysis of adult fish T-maze behavior and larvae fish swimming behavior test}

The zebrafish adult behavior test and larvae swimming behavior test were analyzed using EthoVision XT 13 (Noldus, Wageningen, the Netherlands). We evaluated the velocity, latency, and distance movement of individual zebrafish.

\section{Adult fish muscle endurance swimming performance} Critical swimming speed $\left(U_{\text {crit }}\right)$ is defined as the maximum speed an adult fish can sustain over a period of time [22]. Critical swimming speed was measured in Brett-type swimming tunnel (Loligo Systems, Viborg, Denmark). Fish were not fed for 24 hours before measurements. One fish was selected using a net from the fish tank and placed in the swimming tunnel. After the resting habituation for $5 \mathrm{~min}$, water velocity was gradually increased by $10 \mathrm{~cm} / \mathrm{s}$ in intervals of $5 \mathrm{~min}$ until the fish apparaently showed exhausted swimming performance. The water temperature during experiments was $28 \pm 1{ }^{\circ} \mathrm{C} . U_{\text {crit }}$ was calculated using [22].

$$
U_{\text {crit }}=U_{i}+\left[U_{i i}\left(T_{i} / T_{i i}\right)\right]
$$

where $U_{\mathrm{i}}$ is the highest velocity the fish maintained for the whole $5 \mathrm{~min}(\mathrm{~cm} / \mathrm{s}), U_{\mathrm{ii}}$ is the velocity increase, $T_{\mathrm{i}}$ is the time elapsed at the tired velocity, and $T_{\mathrm{ii}}$ is the time between velocity changes ( $5 \mathrm{~min}$ ).

\section{Analysis of adult fish muscle endurance swimming performance}

Principal component analysis (PCA) was performed using XLSTAT 2014.1 (Addinsoft, NY, USA) to identify the main cause of induced responses and the relationship between these parameters. A biplot was graphed of both the measured parameters and observations.

\section{Chemical treatment for larval fish}

Six chemicals, including L-tyrosine, Taurine, L-carnitine, creatine, Terazosin and ATP, were used to test their effects. Four chemicals: L-tyrosine (All Lines Technology,
PA, USA), Taurine (NIPPON SHINYAKU, Kyoto, Japan), L-carnitine (Sigma-Aldrich, MI, USA) and creatine (Sigma-Aldrich, MI, USA) were dissolved in water at a concentration of $10 \mu \mathrm{M}, 1 \mathrm{mM}, 10 \mu \mathrm{M}$ and $100 \mu \mathrm{M}$ respectively. The concentration were determined according to a previous report on dosage analysis [23]. Terazosin (Selleckchem, TX, USA) was dissolved in Dimethyl sulfoxide (DMSO) at a concentration of $2.5 \mu \mathrm{M}$ following a previous report on dosage analysis [24]. ATP (SigmaAldrich, MI, USA) was dissolved in water at a concentration of $100 \mu \mathrm{M}$. For treatment of the TPM3 transgenic zebrafish model, TPM3 transgenic adult F2 zebrafish were in-crossed to produce the F3 embryos. Following a previous method [23], embryos were immersed with different chemicals all day from 28 hours-post-fertilization (hpf) to 7 days-post-fertilization (dpf) in a $90 \mathrm{~mm}$ dish. The chemical was refreshed every day until $7 \mathrm{dpf}$. Then, zebrafish larvae were transferred into 48-well plates with $1200 \mu \mathrm{L}$ E3 medium for the larvae fish swimming behavior test using DanioVision.

\section{L-Carnitine treatment of larvae fish for one month}

The larva treated L-carnitine from $28 \mathrm{hpf}$ to $7 \mathrm{dpf}$ in $90 \mathrm{~mm}$ dish were continuous treatment from 7 to $30 \mathrm{dpf}$ by immersed in L-carnitine from 20:00 to 08:00 in a $1 \mathrm{~L}$ fish tank. The body weight and body length after treatment were measured for all fish. The swimming tunnel (Loligo Systems, Viborg, Denmark) were used to measure critical swimming speed for the restoration effect of swimming behavior of TPM3 transgenic zebrafish after $\mathrm{L}$-carnitine treatment.

\section{L-carnitine treatment of adult fish from 3 months of age}

TPM3 transgenic zebrafish were immersed in $10 \mu \mathrm{M}$ L-carnitine from 3 months of age from 20:00 to 08:00 for one month. The body weight and body length were measured after treatment. Swimming tunnel (Loligo Systems, Viborg, Denmark) were used to measure the critical swimming speed for the rescue effect of L-carnitine treatment.

\section{Oxygen consumption rate for determination of respiratory function}

Zebrafish embryos develop until 48 hpf. Oxygen consumption rate (OCR) was measured from embryos using a V17 Islet capture plate in the Seahorse XFe24 Analyzer (Agilent, CA, USA). E3 medium was used in wells, and two dechorionated embryos were placed in each well. All measurement cycles for the run were set to $3 \mathrm{~min} \mathrm{mix}$, 2 min wait, and $3 \mathrm{~min}$ measure. Oligomycin was then added followed by 3 measurement cycles. Carbonyl cyanide 4-(trifluoromethoxy)phenylhydrazone (FCCP) was then added followed by another 3 measurement cycles. 
Finally, a mixture of antimycin and rotenone was added followed by 10 measurement cycles. Optimal doses of drugs were optimized separately for TPM3(WT) and TPM3 mutant embryos. Final concentrations of drugs were as follows: TPM3(WT) oligomycin $50 \mu \mathrm{M}$, TPM3 mutant oligomycin $25 \mu \mathrm{M}$, TPM3(WT) FCCP $20 \mu \mathrm{M}$, and TPM3 mutant FCCP $3 \mu \mathrm{M}$. Antimycin and rotenone were added to a final concentration of $2 \mu \mathrm{M}$ for both.

\section{Tissue collection, frozen section, and histological analysis}

Before examination, the fish was anesthetized by $0.025 \%$ buffered MS-222 solution (Sigma-Aldrich, MI, USA) following a previously reported method [25]. We cut the muscle tissue between the anal and caudal fins and placed the muscle tissue in wet gauze. We placed $10 \%$ Tragacanth gum (FUJIFILM Wako Pure Chemical Corporation, Tokyo, Japan) on the well, which was stirred evenly, and formed into a small hill shape. We placed the muscle tissue into the small hill. Then, a beaker filled with $100 \mathrm{~mL}$ 2-methylbutane (Sigma-Aldrich, MI, USA) and placed into liquid nitrogen. We completely submerged the wood with muscle tissue into 2-methylbutane for $1 \mathrm{~min}$. We then transferred muscle tissue to a $-80{ }^{\circ} \mathrm{C}$ freezer immediately. The muscle tissue was sectioned into $8 \mu \mathrm{m}$ slices using Cryostat Microtome CM3050S (Leica, Wetzlar, Germany). Tissue sections were stored in a $-80{ }^{\circ} \mathrm{C}$ freezer, and later stained with Hematoxylin and Eosin stain (HE stain), Gomori's trichrome stain, and nicotinamide adenine dinucleotide dehydrogenase-tetrazolium reductase stain (NADH-TR stain).

\section{Total RNA isolation, qRT-PCR (real-time reverse transcription-PCR)}

The muscle tissue was grounded using Microtube Pellet Pestle Rods (Violet BioScience, Taipei, Taiwan). TRI Reagent $^{\circledR}$ (Sigma-Aldrich, MI, USA) was mixed with sample and homogenized using a homogenizer. 1/5 of chloroform was added, mixed, and later the supernatant containing RNA was transferred to a new Eppendorf. After extracting total RNA from muscle tissue, the total RNA was applied in NucleoSpin ${ }^{\circledR}$ RNA kit (MachereyNagel, Nordrhein-Westfalen, Germany) for the cleanup. RNA was isolated using a NucleoSpin ${ }^{\circledR}$ RNA column (Macherey-Nagel, Nordrhein-Westfalen, Germany) per manufacturer's protocol. Finally, for all the RNA samples, we measured the concentration using a NanoDrop ND-1000 (Thermo Fisher Scientific, MA, USA), which were then stored in a $-80{ }^{\circ} \mathrm{C}$ freezer. Complementary DNA (cDNA) was synthesized using iScript ${ }^{\mathrm{TM}}$ cDNA synthesis kit (BioRad, CA, USA). cDNA was diluted 100-fold for qRT-PCR analysis using SYBR Green (Thermo Fisher Scientific, MA, USA). Primers for qRT-PCR are listed in Additional file 1: Table S1.

\section{Next generation sequencing analysis}

Next generation sequencing experiment was performed by NHRI Genomics Core Facility. Sequencing libraries $1 \mu \mathrm{g}$ total RNA were generated using RNA HyperPrep kit with RiboErase (Kapa Biosystems, Pleasanton, CA, USA), and sequenced on NovaSeq 6000 instrument (Illumina, San Diego, CA, USA). All RNA sample preparation procedures were carried out according to the Illumina's official protocol. Agilent's SureSelect Strand-Specific RNA Library Preparation Kit was used for library construction followed by AMPure XP beads (Beckman Coulter, USA) size selection. Illumina's bcl2fastq program v2.20 used for basecalling. De-multiplexed sequencing data was processed with Trimmomatic (version 0.36) to remove any adapter and poor-quality sequences with a slidingwindow approach. Resulting read pairs were aligned with a HISAT2 aligner against zebrafish GRCz11 reference genomes (Ensembl version 99) to obtain reads count and fragments per kilobase per million (FPKM) data of genes and transcripts.

The expression analysis settings were as follows: Genelevel fold change $<-2$ or $>2$, gene-level $p$-value $<0.05$, ANOVA method: Ebayes. The gene ontology analysis was performed using gene ontology analysis via WEB-based GEne SeT AnaLysis Toolkit (WebGestalt [26], http:// www.webgestalt.org/), pathway analyses were performed by NetworkAnalyst (http://www.networkanalyst.ca/), and activated pathways were selected and matched according to the Kyoto Encyclopedia of Genes and Genomes (KEGG) database.

\section{Statistical analysis}

Data were collected and analyzed using Excel (Microsoft, WA, USA) and GraphPad Prism 9 software (GraphPad Software, CA, USA). Individual tests were described in the main text. All the statistical analyses were conducted using two-tailed Student's $t$-test. All data are expressed as mean \pm standard error of the mean (SEM). A $P$-value less than 0.05 was considered statistically significant. For all figures: NS, $P>0.05$; *, $P \leq 0.05$; **, $P \leq 0.01$; ***, $P \leq 0.001$; ***** $P \leq 0.0001$.

\section{Results}

TPM3 mutant transgenic fish shows abnormal appearance and lower survival rate

Three transgenic fish expressing human TPM3(WT), TPM3(E151A), and TPM3(E151G) under the promoter of myosin light chain 2 ( $M L C 2$ ) were generated as described previously [17] with details in materials and methods. The mRNA level of TPM3 transgenic zebrafish were examined by qRT-PCR and confirmed all three TPM3 transgenic zebrafish expressed human TPM3 gene (Additional file 2: Fig. S1A). When compared to the non-transgenic 
(See figure on next page.)

Fig. 1 Appearance, survival rate, X-ray images and histopathological examinations of muscle in TPM3 transgenic zebrafish. a Appearance of F0 adult TPM3 transgenic fish at 6.5 months of age and F1 adult TPM 3 transgenic fish at 1.5 months of age. Scale bar is $1 \mathrm{~cm}$. b Representative images of the F1 larvae TPM3 transgenic fish. c Expression level of human TPM3 mRNA in TPM3 transgenic fish compared to non-transgenic control AB(WT). Orange bar represents $A B(W T)$, red bar denotes four TG lines of TPM3(WT), blue color indicates four TG line of TPM3(E151A), green plot indicates four TG lines of TPM3(E151G). d Phenotypical analysis of the F1 larvae TPM3 transgenic fish, crooked body were indicated as grey color, TPM3 mutant lines exhibited higher percentage of abnormaility compared to TPM3(WT) e Survival rate of the F1 larva of TPM3 transgenic fish, TPM3 mutant lines exhibited lower survival rate compared to TPM3(WT). $\mathbf{f}$ X-ray images of skeleton alignment for adult AB(WT), TPM3(WT), TPM3(E151A), and TPM3(E151G). Zebrafish have 31 vertebrae in male and female fish. AB(WT) and TPM3(WT) exhibited normal skeleton alignment. TPM3(E151A) TG2 and TG4 showed abnormal skeleton alignment. All four independent lines of TPM3(E151G) transgenic zebrafish displayed some levels of abnormal skeleton alignment. Scale bar is $1 \mathrm{~cm}$. $\mathbf{g}$ Hematoxylin and Eosin stain (HE) stain, modified Gomori trichrome stain, and NADH-TR stains of adult $\mathrm{AB}(\mathrm{WT})$ and adult F1 TPM3 transgenic zebrafish at 50X, scale bar is $200 \mu \mathrm{m}$. $\mathbf{h}$ Representative images of three staining at $400 \mathrm{X}$, scale bar is $50 \mu \mathrm{m}$

$\mathrm{AB}(\mathrm{WT})$ control fish, overexpressed TPM3(WT) or TPM3(E151A) mutant, those fish displayed normal appearance, however, overexpressed E151G mutant TPM3, the fish showed abnormal appearance with no tail as early as F0 (Fig. 1a). This abnormal morphology was inherited to F1 adult fish (Fig. 1a). The F1 larvae from TPM3(E151G) and TPM3(E151A) transgenic fish also exhibited a higher proportion of abnormalities (Fig. 1b). Since this de novo mutation is heterogeneous in patient, it is genetic dominant. To clarify whether the observed defect is really due to the expression of the TPM3 gene rather than other nonspecific effects, we have measured the human TPM3 RNA expression via qRT-PCR analysis using RNAs from various transgenic strains and showed the transgenic fish indeed overexpression human TPM3 gene (Fig. 1c).

Among four independent lines of TPM3(E151G) fish, $9.8 \%$ of TG1, $5.9 \%$ of TG2, and $86.7 \%$ of TG3 displayed a crooked body appearance. Even for the TPM3(E151A) fish, $4.2 \%$ of TG1 and $68 \%$ of TG2 had crooked body appearance (Fig. 1d). The survival rate of F1 larvae was affected by the TPM3 mutations. In general, the survival rates of both TPM3 mutants were decreased compared to TPM3(WT), and TPM3(E151G) had a much lower survival rate than TPM3(E151A) (Fig. 1e).

\section{TPM3(E151G) exhibits more severe vertebrae abnormalities than TPM3(E151A)}

As the mutant TPM3 transgenic zebrafish showed abnormal body appearances, we further examined the skeleton imaging of the TPM3 transgenic zebrafish using a digital $\mathrm{X}$-ray detector [19]. The non-transgenic $\mathrm{AB}(\mathrm{WT})$ control fish has compared to the wildtype or mutant TPM3 transgenic fish for the skeleton, ten $\mathrm{AB}(\mathrm{WT})$ fish were all normal (Fig. 1f). Four independent lines of TPM3(WT) F1 adult fish all had normal aligned vertebrae (Fig. 1f). In the two independent TG2 and TG4 lines of TPM3(E151A) F1 fish, we observed four out of seven fish (57.1\%) and two out of seven fish $(28.5 \%)$ respectively with malformations in the vertebrae (Fig. 1f). All four independent lines of
TPM3(E151G) F1 adult fish exhibited skeleton abnormalities, with malformations in two out of eight (25\%), four out of seven (57.1\%), two out of four (50\%), and two out of eight fish (25\%) for TG1 to TG4, respectively (Fig. 1f). The X-ray imaging results showed that overexpression of wildtype TPM3 does not contribute to pathology, TPM3 mutations produce a deformity in the precaudal vertebrae in most fish, and that in TPM3(E151G) was more severe than in TPM3(E151A).

\section{TPM3(E151G) displays congenital fiber type disproportion and TPM3(E151A) resembles nemaline myopathy}

Muscle biopsy is an important diagnostic resource for confirming CM. We used the cryosection and histochemical staining to examine the muscle specimens of TPM3 transgenic zebrafish in F1 adults, the non-transgenic $\mathrm{AB}(\mathrm{WT})$ control has compared to TPM3 transgenic fish for the muscle pathology. Both $\mathrm{AB}(\mathrm{WT})$ and TPM3(WT) fish all had normal muscle cell from all three staining. By HE stain, we observed that the muscle fibers of TPM3(WT) were arranged neatly and consistent, TPM3(E151A) fish shows smaller-sized fibers and TPM3(E151G) shows variation in fiber size (Fig. 1g, h). TPM3(E151A) fish had rod-like structures in the muscle fibers resembled nemaline myopathy on Gomori trichrome stain (Fig. 1g, h). In zebrafish, type 1 fiber is usually smaller than type 2 fibers. Using NADH-TR stain under low magnification (50X), the TPM3(WT) fish have both type 1 (darker) and type 2 (paler) fibers similar to non-transgenic control $\mathrm{AB}$ (WT) (Fig. 1g). TPM3(E151A) transgenic fish also showed both types. On the other hand, TPM3(E151G) has increased smallersized and darkly stained fibers (Fig. 1g), the muscle fibers of TPM3(E151G) displayed small and dark-stained type 1 muscle fibers and disproportionate arrangement, similar to CFTD (Fig. 1g, h).

To further elucidate whether expression of WT or mutant TPM3 caused fiber type conversion, we performed qRT-PCR for the expression level of type 1 and type 2 heavy chain. The myosin heavy chain isoform 


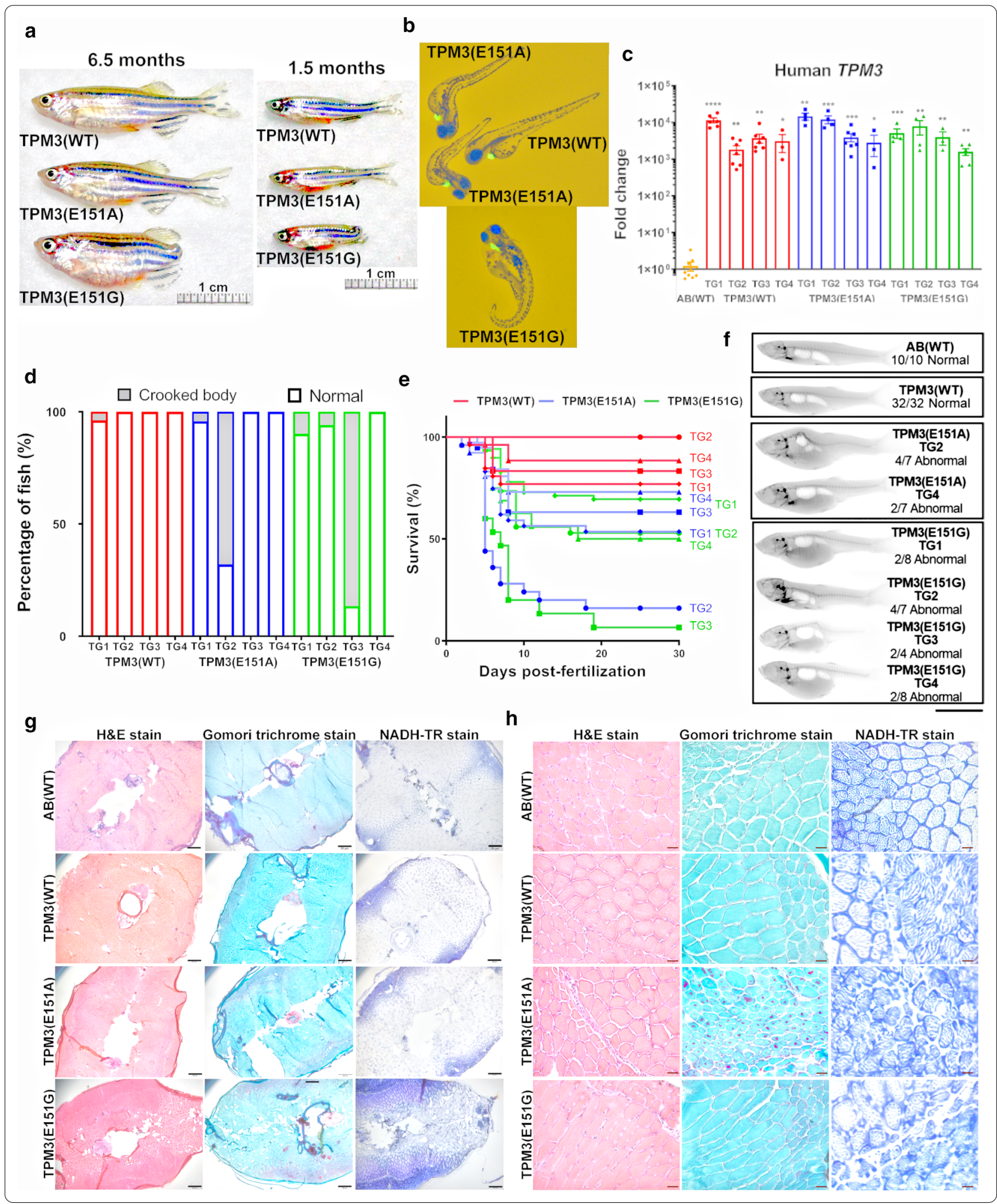




\begin{abstract}
(See figure on next page.)
Fig. 2 T-maze behavior test for adult TPM3 transgenic zebrafish, and the swimming velocity of larval TPM3 transgenic zebrafish. a The swimming velocity of F1 larva TPM3(WT) transgenic fish compared to non-transgenic $A B(W T)$ control fish during the five days of testing. Red plot denotes TPM3(WT) fish $(n=28)$, orange plot represents $A B(W T)$ fish $(n=12)$. There is no significant differences between TPM3(WT) and AB(WT). b The swimming velocity of F1 larva TPM3 transgenic fish at $7 \mathrm{dpf}$. Orange plot represents AB(WT) $(n=12)$, red plot denotes four TG lines of TPM3(WT) ( $n=8$ for each line), blue plot indicates four TG line of TPM3(E151A) ( $n=8$ for each line), green plot indicates four TG lines of TPM3(E151G) ( $n=8$ for each line). Four lines of TPM3(E151G) and TG2 of TPM3(E151A) significantly swim slower compared to AB(WT). c The swimming velocity of F0 adult TPM3 transgenic zebrafish during the five days of testing. Red plot represents TPM3(WT) $(n=10)$, blue plot indicates TPM3(E151A) $(n=10)$, green plot indicates TPM3(E151G) $(n=10)$. $\mathbf{d}$ Latency for memory test of F0 adult TPM3 transgenic zebrafish during the five days of testing. TPM3(WT), TPM3(E151A), and TPM3(E151G) are shown in red, blue, and green bars, respectively. e The swimming velocity of F1 adult TPM3 transgenic zebrafish during the five days of testing. Red plot represents TPM3(WT) TG1 $(n=10)$, blue plot denotes TPM3(E151A) TG1 $(n=10)$, green plot indicates TPM3(E151G) TG1 ( $n=10)$, brown plot is TPM3(E151G) TG1 abnormal appearance $(n=6)$, and pink plot is TPM3(E151G) TG2 $(n=10)$. $\mathbf{f} L a t e n c y$ for memory test of F1 adult TPM3 transgenic zebrafish during the five days of testing. TPM3(WT) TG1, TPM3(E151A) TG1, TPM3(E151G) TG1, TPM3(E151G) TG1 abnormal appearance, and TPM3(E151G) TG2 are shown in red, blue, green, brown, and pink bars, respectively. Statistical significance was determined in comparison with $\mathrm{AB}(\mathrm{WT})$ and was determined using a $t$-test, ${ }^{*} 0.01<P \leq 0.05 ;{ }^{* *} 0.001<P \leq 0.01 ;{ }^{* * *} 0.0001<P \leq 0.001 ;{ }^{* * * * P} \leq 0.0001$
\end{abstract}

for type 1 muscle is encoded by $m y h 7$, and type $2 \mathrm{~A}$ is encoded by $m y h z 2$ in zebrafish [27], we measured the expression of $m y h 7$ and $m y h z 2$ for the $\mathrm{AB}(\mathrm{WT})$, wildtype and mutant TPM3 transgenic fish. Both TPM3(WT) and TPM3(E151A) expressed higher level of type 1, and lesser type 2 myosin heavy chain compared to $\mathrm{AB}(\mathrm{WT})$, so there is fiber type conversion. On the other hand, TPM3(E151G) expressed lower levels of both type 1 and type 2 myosin heavy chain (Additional file 2: Fig. S1A, B). So, it seems like there is no fiber type conversion in TPM3(E151G), but generally losing both muscle type.

\section{TPM3 mutant transgenic fish display reduced swimming ability compared to TPM3(WT) fish}

To examine whether the affected muscle development in TPM3 mutants may lead to swimming disability, the swimming velocity and latency of TPM3 transgenic fish were measured by using DanioVision for larva and T-maze for adult zebrafish. The $\mathrm{AB}(\mathrm{WT})$ fish control were compared to the wildtype or mutant TPM3 transgenic fish for the swimming velocity, and there is no difference between $\mathrm{AB}(\mathrm{WT})$ and wildtype TPM3 transgenic fish larva (Fig. 2a); the four independent lines of TPM3(E151G) at $7 \mathrm{dpf}$ all exhibited significant lower velocity compared to $\mathrm{AB}(\mathrm{WT})$ fish (Fig. 2b). TPM3(E151A) TG2 swimming velocity was also significantly lower compared with the TPM3(WT) fish at $7 \mathrm{dpf}$ (Fig. 2b). In addition, we measured the swimming velocity of 3 to $6 \mathrm{dpf}$, and found that TPM3(E151G) larvae exhibited lower velocity compared to $\mathrm{AB}(\mathrm{WT})$ (Additional file 2: Fig. S1C-F). Whereas the F0 TPM3(E151G) adult fish after day 3 to day 5 exhibited significantly lower swimming velocity compared to TPM3(WT), we found no difference between TPM3(WT) and TPM3(E151A) (Fig. 2c). However, regarding the latency, TPM3(E151G) and TPM3(E151A) required significantly longer time to find the target compared with TPM3(WT) after day 3 to day 5 (Fig. 2d). We also examined those in the F1 adult of the TPM3 transgenic fish, and found that both TPM3(E151A) and TPM3(E151G) F1 adults still exhibited significantly lower swim velocity compared with TPM3(WT) F1 adult (Fig. 2e). We also found the two groups of TPM3(E151G) TG1 line, with or without tails (abnormal), all swam slower than TPM3(WT). In latency, all F1 adult fish of the TPM3(E151G) and TPM3(E151A) required significantly longer time to find the target compared with TPM3(WT) F1 adult (Fig. 2f). The data above indicate TPM3 mutant transgenic fish display reduced swimming ability. The swimming behavior of TPM3 transgenic fish are shown in Additional file 3: Movie S1.

\section{TPM3 mutant transgenic fish discloses weaker muscle endurance}

The T-maze swimming test may combine learning and memory in the swimming behavior and may not be suitable to only represent the muscle strength. To precisely examine the strength of the muscles, critical swimming speed $\left(U_{\text {crit }}\right)$ to measure muscle endurance in a swimming tunnel for a total of 13 groups of F1 adult zebrafish including $\mathrm{AB}(\mathrm{WT})$ zebrafish, four independent lines for TPM3(WT), TPM3(E151A), and TPM3(E151G) was determined. A non-transgenic $\mathrm{AB}(\mathrm{WT})$ fish control has compared to the wildtype TPM3 transgenic fish for the critical swimming speed, no differences in muscle endurance between $\mathrm{AB}(\mathrm{WT})$ and TPM3(WT) adult fish were detected (Fig. 3a). Importantly all four independent lines of TPM3(E151G) exhibited significantly debilitated muscle endurance compared with $\mathrm{AB}(\mathrm{WT})$ (Fig. 3a). Two independent lines TG2 and TG4 of TPM3(E151A) showed significantly weaker muscle endurance compared with $\mathrm{AB}(\mathrm{WT})$ (Fig. 3a). We showed zebrafish swimming near $U_{\text {crit }}$ in the end of Additional file 3: Movie S1. 

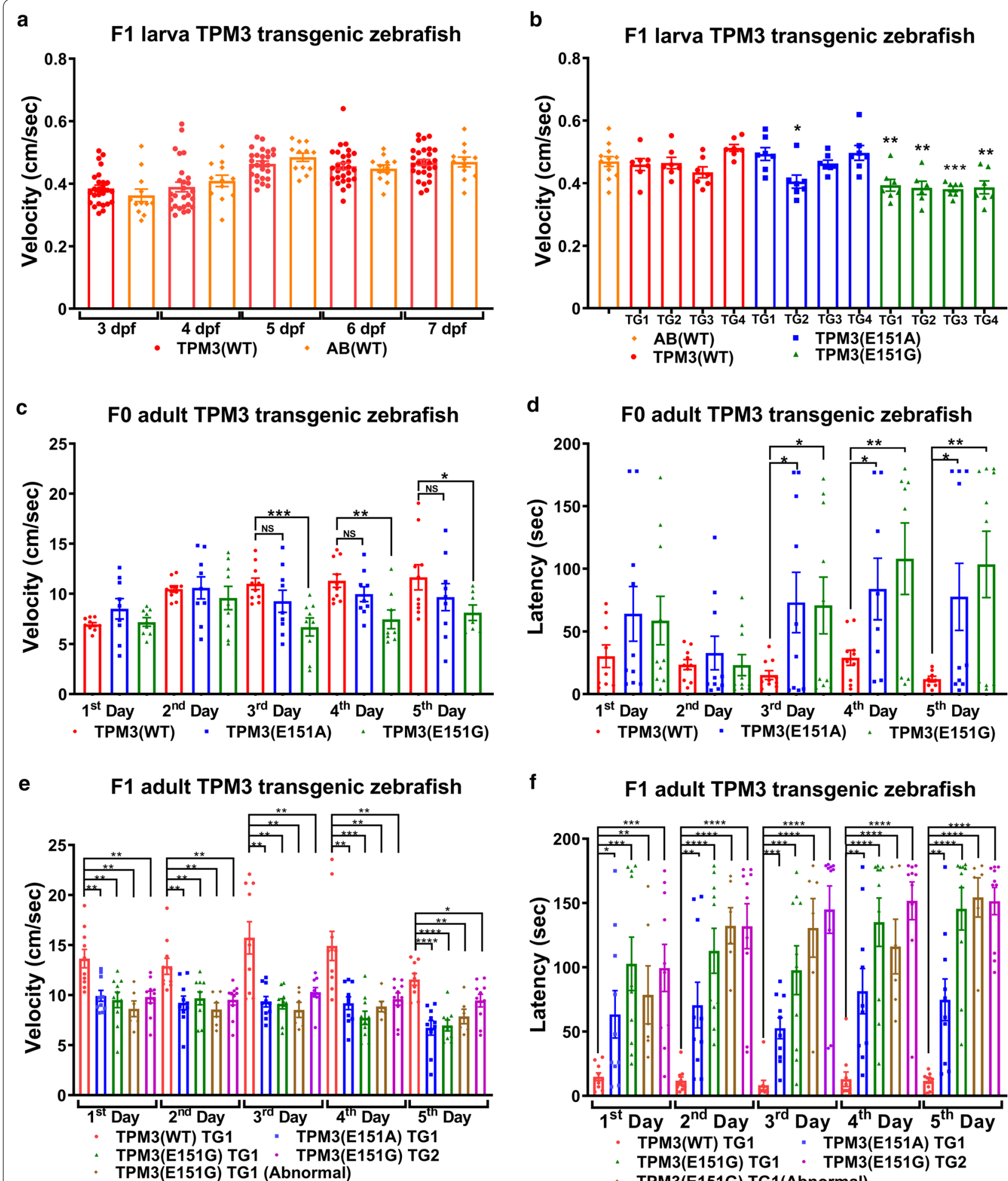

f

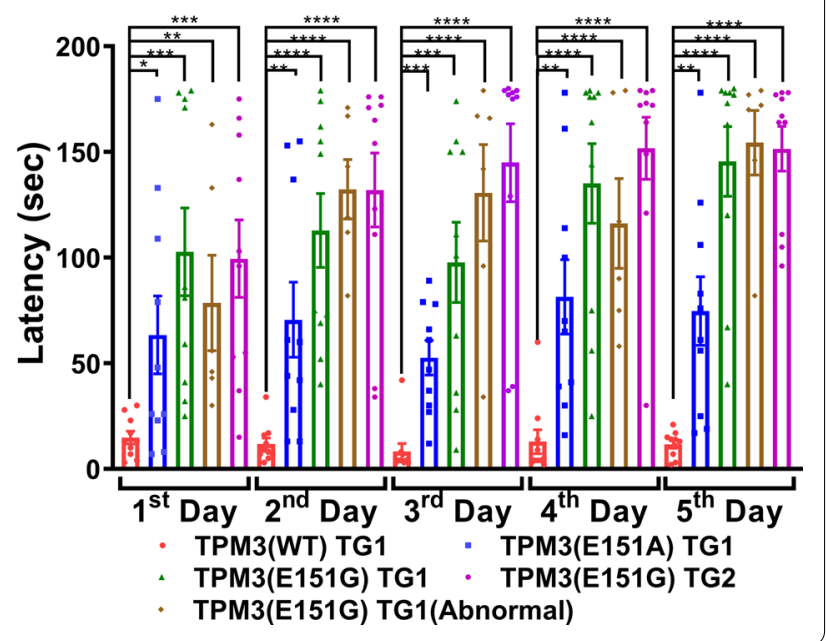

We wondered whether the body weight or length of these 13 groups might have resulted in the difference in muscle endurance test; only TPM3(WT) TG3, and
TPM3(E151G) TG1 and TG2 had significantly lower body weight compared to $\mathrm{AB}(\mathrm{WT})$ (Fig. 3b). After standardizing the length, some of the fish, including 

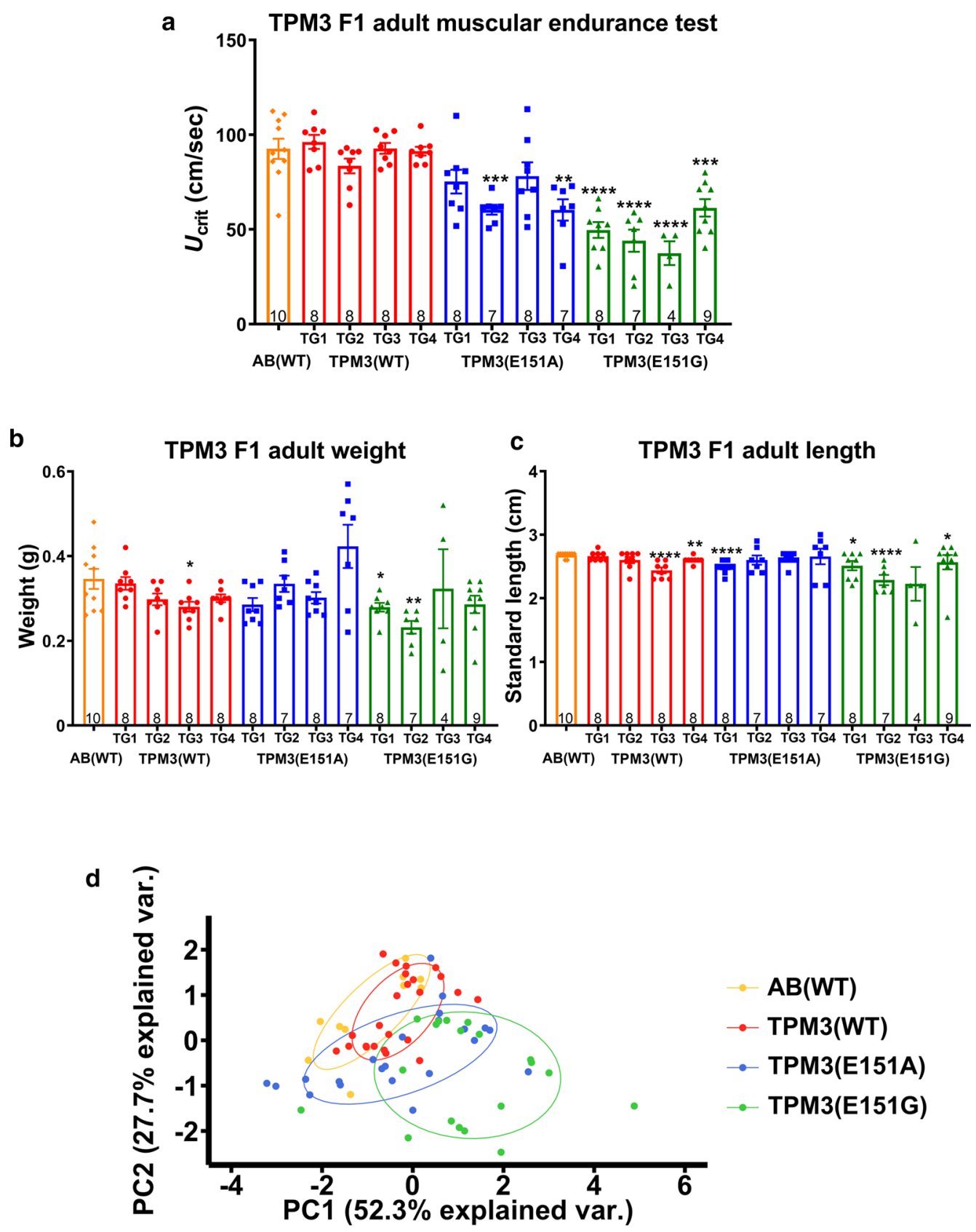

Fig. 3 Muscle endurance test for adult F1 TPM3 transgenic zebrafish. a Critical swimming speed $\left(U_{\text {crit }}\right)$ was used to measure the muscle endurance using a swimming tunnel. The statistical significance was calculated in comparison with $A B(W T)$. The orange plot is $A B(W T)(n=10)$, red plot is the four TG lines of TPM3(WT) (TG1, $n=8 ; \mathrm{TG} 2, n=8 ; \mathrm{TG} 3, n=8 ; \mathrm{TG} 4, n=8)$, blue plot is the four TG lines of TPM3(E151A) (TG1, $n=8 ; \mathrm{TG} 2, n=7$; $\mathrm{TG} 3, n=8 ; \mathrm{TG} 4, n=7)$, green plot is the four TG lines of TPM3(E151G) (TG1, $n=8 ; \mathrm{TG} 2, n=7 ; \mathrm{TG} 3, n=4 ; \mathrm{TG} 4, n=9)$. $\mathbf{b}$ The body weight of the 13 groups. c The standard length of the 13 groups. $\mathbf{d}$ Principal component analysis of endurance test for adult F1 TPM3 transgenic zebrafish. The first principal component (PC) accounted for $52.3 \%$ and the second PC for $27.7 \%$ of the variance. The orange plot is AB(WT) $(n=10)$, red is TPM3(WT) $(n=24)$, blue is TPM3(E151A) $(n=22)$, and green is TPM3(E151G) $(n=19)$. Statistical significance was determined using a t-test, ${ }^{*} 0.01<P \leq 0.05$; ${ }^{* *} 0.001<P \leq 0.01 ;{ }^{* * *} 0.0001<P \leq 0.001 ;{ }^{* * *} P \leq 0.0001$

TPM3(WT) TG3 and TG4, TPM3(E151A) TG1, and TPM3(E151G) TG1 and TG2, were significantly shorter, whereas TPM3(E151G) TG4 was significantly longer compared with $\mathrm{AB}(\mathrm{WT})$ (Fig. 3c). Therefore, there is no correlation between either body weight or length and muscle endurance. 
Four variation factors, critical swimming speed $\left(U_{\text {crit }}\right)$, sex, body weight, and standard length, were analyzed by using principal component analysis. When two principle factors were selected, the total explanatory power was $80 \%$. The first principal component (PC) and the second PC accounted for $52.3 \%$ and $27.7 \%$ of the variance, respectively. When the two PC factors represented all the factors, only $20 \%$ of the variation was ignored. We observed that $\mathrm{AB}(\mathrm{WT})$ and TPM3(WT) almost overlap, which we interpreted as the same group. TPM3(E151G) is separated from $\mathrm{AB}(\mathrm{WT})$ and TPM3(WT), which is obviously different from the $\mathrm{AB}(\mathrm{WT})$ and TPM3(WT) group (Fig. 3d).

\section{L-Carnitine specifically improves the swimming velocity of TPM3(E151G) larvae}

Based on the results above, we found that TPM3(E151G) mutant zebrafish exhibited weaker muscle endurance and slower swimming behavior, resembling human patients. As no treatment exists for $\mathrm{CM}$, we used the TPM3(E151G) zebrafish to screen for potential therapeutic drugs. TPM3 transgenic fish (F3) were treated with five chemicals (L-carnitine, L-tyrosine, taurine, creatine, and terazosin) from 28 h-post-fertilization (hpf) to 7 day-post-fertilization (dpf), and we measured the swimming velocity by using DanioVision. Amazingly, we found that only L-carnitine significantly improved the swimming velocity of TPM3(E151G) larvae, but the other four chemicals did not (Fig. 4a). In addition, L-carnitine treatment is specific to TPM3(E151G) to improve the swimming velocity, since the swimming speeds of TPM3(E151A) (Fig. 4b) and AB(WT) (Fig. 4c) fish were not altered by L-carnitine treatment. The results indicate that L-carnitine specifically enhances the swimming velocity of TPM3(E151G) larvae, but not the swimming velocity of TPM3(E151A) and $\mathrm{AB}(\mathrm{WT})$ larvae.

L-Carnitine can store and regulate the supply of ATP to enhance muscle endurance [6]. Thus we examined whether supplementation with ATP can produce a similar effect. As shown in Fig. 4d, ATP supplementation also significantly increased the swimming velocity of TPM3(E151G) F3 larvae but had no beneficial effect to TPM3(WT) F3 larvae (Fig. 4e).

To confirm muscle endurance could be restored by L-carnitine, the TPM3 transgenic fish (F3) were treated with L-carnitine from $28 \mathrm{hpf}$ for 30 days and measured for the muscle endurance using a swimming tunnel at 30 dpf. As expected, the muscle endurance of TPM3(E151G) fish was restored by L-carnitine treatment (Fig. 4f). One month of L-carnitine treatment also increased the weight (Fig. 4g) and length of the TPM3(E151G) transgenic fish (Fig. 4h). Using principal component analysis (PCA), we observed that TPM3(WT) and
TPM3(E151G) + L-carnitine overlapped, indicating L-carnitine effectively restored the TPM3(E151G) muscle endurance to resemble the muscle endurance of TPM3(WT) (Fig. 4i). However, one month of L-carnitine treatment on TPM3(E151G) transgenic zebrafish starting from the age of three months failed to restore the muscle endurance (Additional file 4: Fig. S2), implying the treatment must be applied early during muscle development. We applied PCA analysis for $\mathrm{AB}(\mathrm{WT})$ and TPM3(WT) for all the fish in this study, and found there is no difference between them (Fig. 4j). This data indicated that overexpression of wildtype TPM3 does not contribute to pathology, including fish survival, swimming velocity, $\mathrm{T}$-maze, and muscle endurance.

\section{Reductions of mitochondria basal respiration and ATP production and muscle pathology in TPM3(E151G) mutation transgenic fish can be rescued by L-carnitine treatment}

Since L-carnitine and ATP treatments both restored the muscle endurance of TPM3(E151G) transgenic fish, we wondered whether mitochondria respiration and ATP production were impaired in the TPM3(E151G) transgenic fish. The TPM3(WT), TPM3(E151A), TPM3(E151G), and L-carnitine-treated TPM3(E151G) transgenic fish were subjected to Seahorse XF Extracellular Flux Analyzer (Agilent Technologies, Santa Clara, CA, USA), and the mitochondria respiration data revealed TPM3(E151G) mutation caused significant decreases in basal respiration and ATP production (Fig. 5a-d).

To understand the muscle pathology changed after L-carnitine treatment, we performed NADH-TR stain for the one month of $\mathrm{L}$-carnitine treated TPM3 transgenic fish. Both the muscle volume and fiber size were increased in L-carnitine-treated TPM3(E151G) transgenic fish, and they were similar to TPM3(WT) (Fig. 5e). To further understand the muscle fiber type composition after L-carnitine treatment, we examined the expression of type I (myh7) and type II (myhz2) myosin fiber from TPM3 transgenic fish (F3) treated with L-carnitine from $28 \mathrm{hpf}$ to $7 \mathrm{dpf}$, and found that the expression level of $m y h 7$ and $m y h z 2$ in TPM3(E151A) and TPM3(E151G) was significantly reduced. After L-carnitine treatment, the expression of $m y h 7$ and $m y h z 2$ is significantly recovered to the same as TPM3 (WT) (Fig. 5f, g). The effect of L-carnitine can also be found in both body weight and length. After feeding L-carnitine for one month, the weight and body length of TPM3(E151G) increased significantly indicated the fish became healthier (Additional file 5: Fig. S3). 


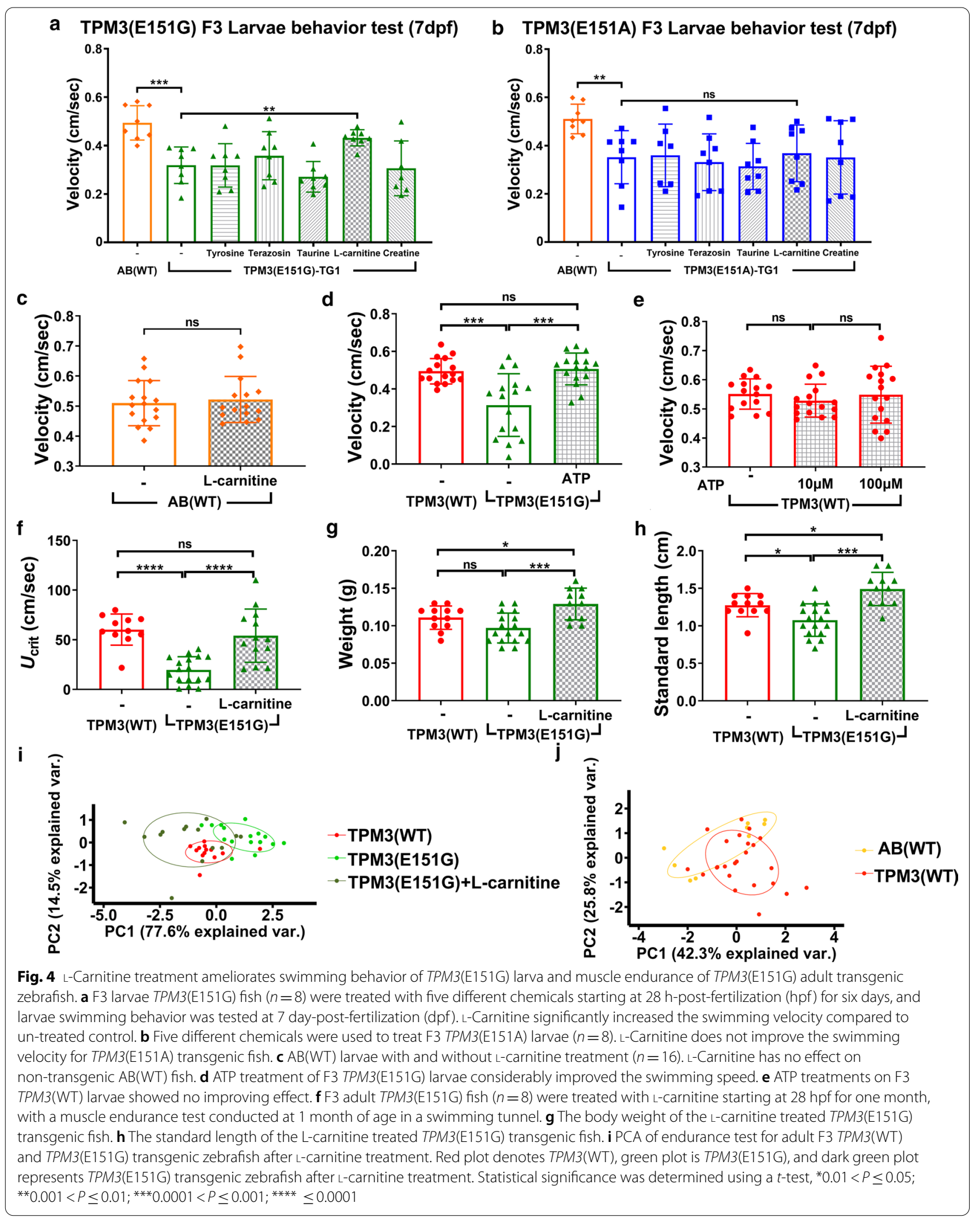


(See figure on next page.)

Fig. 5 L-Carnitine treatment increased mitochndria activity and the type I and type II myosin light chain expression of TPM3(E151G) larva. Mitochondrial respiration of TPM3 transgenic zebrafish via seahorse. a Schematic and oxygen consumption rate (OCR). OCR was measured under basal conditions followed by the sequential addition of oligomycin $(0.5 \mu \mathrm{M})$, Carbonyl cyanide-4-(trifluoromethoxy) phenylhydrazone (FCCP) $(4 \mu \mathrm{M})$, rotenone $(1 \mu \mathrm{M})$, or antimycin A $(1 \mu \mathrm{M})$. b Basal respiration, c ATP production, and $\mathbf{d}$ proton leak of OCR measurement. e NADH-TR stains of TPM3(WT), TPM3(E151G) and one month of L-carnitine treated TPM3(E151G) transgenic zebrafish at 400X, scale bar is $50 \mu \mathrm{m}$. $\mathbf{f}$ the expression level of myh7 and $\mathbf{g}$ myhz2 from TPM3 transgenic fish (F3) treated with L-carnitine from $28 \mathrm{hpf}$ to $7 \mathrm{dpf}$ cpmpared to TPM3(WT). Statistical significance was determined using a $t$-test, ${ }^{*} 0.01<P \leq 0.05 ;{ }^{* *} 0.001<P \leq 0.01 ;{ }^{* * *} 0.0001<P \leq 0.001$; ${ }^{* * * *} P \leq 0.0001$

\section{Next-generation sequencing discloses genes in ion channels and pathways dysregulated by TPM3 mutation which can be reverted by L-carnitine treatment} To understand the molecular mechanism of TPM3related $C M$, we extracted the RNA from the muscle specimens of adult TPM3 mutant and WT transgenic fish and performed next-generation sequencing (NGS) analysis. Downregulation of genes in two TPM3 mutant fish compared to TPM3(WT) were identified as involved in anatomy structure development, cell differentiation, and cell morphogenesis from the expression profiling of the muscle specimens (Additional file 6: Fig. S4A). Genes involved in anatomy structure development were also downregulated in muscle from embryos (Additional file 6: Fig. S4B). The fold changes from TPM3(E151G) were found to be much more lower than TPM3(E151A) in adults (Additional file 6: Fig. S4A), consistent with the recorded lower muscular endurance in TPM3(E151G). Comparing the differentially expressed genes (DEGs) from embryos and adults and verified with qRT-PCR, we found genes involved in anatomy structure development were both downregulated in embryos and adults (Additional file 6: Fig. S4C), and could be rescued by L-carnitine treatment (Additional file 6: Fig. S4D, Additional file 7: Fig. S5). Among them, caldesmon $1 b$ (cald1b) exhibits actin binding activity and myosin heavy chain binding activity.

We further analyzed the overlapped genes between TPM3(E151G) mutant and TPM3(E151G) with L-carnitine treatment, and found 488 overlapped genes which were downregulated more than two-fold in TPM3(E151G) and upregulated in TPM3(E151G) with L-carnitine treatment, but only 9 overlapped genes whose expressions were upregulated in TPM3(E151G) and downregulated in TPM3(E151G) with L-carnitine treatment (Fig. 6a,b). Using gene ontology analysis via WebGestalt [26], we found those overlapped genes downregulated in TPM3(E151G) and rescued by L-carnitine treatment involved in ion transmembrane transporter, especially potassium, calcium and sodium ion channels were enriched (Fig. 6c, d), the differentially expressed genes involved in anatomy structure development and ion channels were validated using qRT-PCR (Fig. 6e). Interestingly, sodium channel mutations has been reported to associate with severe myopathy with hypotonia hypokinesia or classical CM [28]. The results reveal some potential target genes in ion channels and pathways as the molecular mechanism of the TPM3 mutant related $\mathrm{CM}$, which may be used for further therapeutic targets.

\section{Ingenuity pathway analysis (IPA) revealed differential dysregulated pathways, functions and upstream regulators between TPM3(E151A) and TPM3(E151G)}

From pathological findings, muscle functional analyses and pharmacological responses, TPM3(E151A) and TPM3(E151G) show quite different phenotypes, from gene expression analysis, TPM3(E151A) and TPM3(E151G) also display differential gene expression, we have carry out Ingenuity Pathway Analysis (IPA) to find out the enriched pathways, diseases and functions as well as upstream regulators. In the embryos, TPM3(E151A) and TPM3(E151G) share few pathways, for example they both downregulated the synaptogenesis signaling pathways, GNRH signaling and insulin secretion signaling (Fig. 7). However, TPM3(E151A) dysregulated many different canonical pathways, upregulated Tol-like receptor signaling pathways and $\mathrm{T}$ cell exhaustions signaling pathways are only observed in TPM3(E151A) (Fig. 7a). In the upstream regulators, the TPM3(E151A) and TPM3(E151G) embryos share many similar upstream regulators, for example they both downregulated the IFNA2, IRF3, PI3K, Creb, STAT1, CEBPB, and L-carnitine treatment can reverse those changes (Fig. $7 \mathrm{~b}$ ). The biological changes among TPM3(E151A) and TPM3(E151G) are also different (Fig. 7c). E151G mutant downregulated the fatty acid metabolism and synthesis of fatty acid which can be reverse by L-carnitine treatment (Fig. $7 \mathrm{~d}$ ), but it was not seen in E151A. This can explain why the L-carnitine only can rescue the TPM3(E151G) but not TPM3(E151A) transgenic fish. However, release of $\mathrm{Ca} 2+$, transport of molecule and hydrolysis of phospholipid are only downregulated in TPM3(E151A). Therefore, the TPM3(E151A) might need the $\mathrm{Ca} 2+$ transporter activator to rescue its muscle strength.

The TPM3(E151A) and TPM3(E151G) adult share some pathways, but, TPM3(E151G) exhibited more severe downregulated canonical pathways than in 


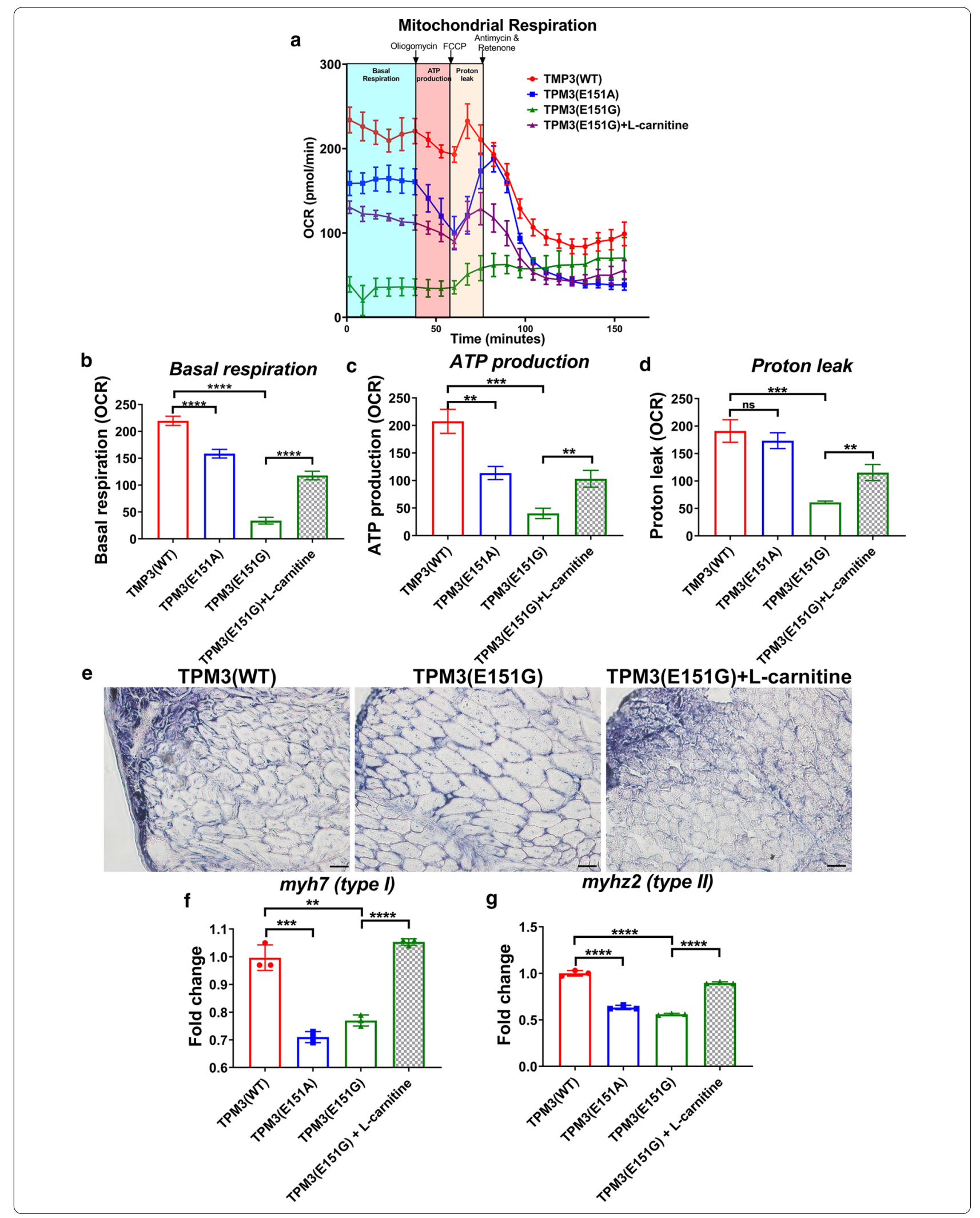




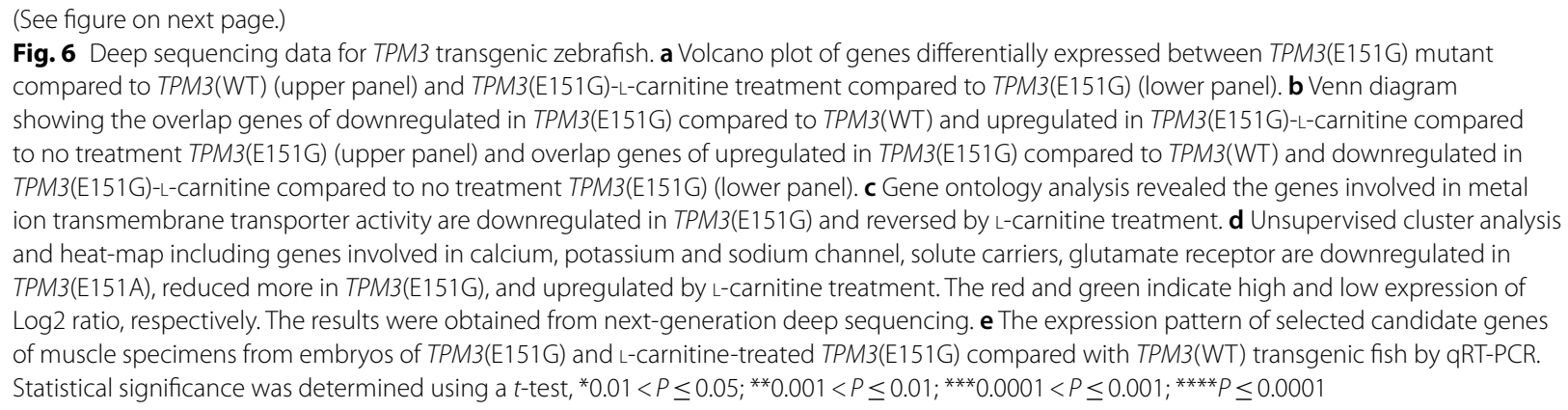

TPM3(E151A) (Fig. 8a). The TPM3(E151A) and TPM3(E151G) adult share some biological functions, such as transport of molecule, transport of cation, transport of ion. However, the majority biological functions are differentially regulated in TPM3(E151G) and TPM3(E151A) (Fig. 8b). Both the genes' expression dysregulated in TPM3(E151A) and TPM3(E151G)-adult involved in movement disorders (Fig. 8c, d).

\section{Discussion}

In this study, we have generated transgenic zebrafish expressing wild type, E151A and E151G TPM3 in muscles to study the pathogenicity of TPM3(E151G)-related CM. TPM3(E151G) fish displayed lower survival rate, severe skeletal abnormalities, and presented fiber type disproportion and exhibited weaker muscle endurance. Furthermore, we identified L-carnitine specifically improved the swimming velocity of TPM3(E151G) larvae and increased basal respiration and ATP production in mitochondria. The RNA-seq analysis from the muscles of TPM3(E151G) larvae revealed the downregulated genes involved in pathways of sodium, potassium and calcium ion channels which their downregulation can be reverted by L-carnitine treatment. TPM3(E151G) is a new novel mutation that has not been reported, and has much severe phenotypes than TPM3(E151A) mutation previously reported. Our study provides the evidence and prove that human TPM3(E151G) mutation was pathogenicity in TPM3-related CM by using zebrafish.

For the behavioral test, we started with the T-maze to measure the swimming velocity and memory test related to learning in our TPM3 transgenic zebrafish. In ethology, the T-maze is a simple maze used for animal cognitive experiments. The T-maze is widely used to measure the spatial memory and learning tasks in laboratory animals, such as rat or zebrafish. Zebrafish has quickly become an important model for studying vertebrate neural development [29]. Due to its simple test for the function of learning, the T-maze has been widely used to study drugs and toxins that affect memory in zebrafish [30]. As in other studies, the TPM3(WT) zebrafish can be trained, indicating that TPM3(WT) zebrafish has demonstrated good learning ability and memory in the T-maze test. However, TPM3 mutant zebrafish differed considerably from TPM3(WT), implying TPM3 mutant zebrafish might experience memory deficits and are unable to learn. To eliminate the influence of learning ability, we used a swimming tunnel to test exact muscle endurance without learning ability. The swimming tunnel uses different water flowing velocities to keep the fish in a state of swimming to measure the muscle endurance. We proved TPM3 mutation fish did reduce muscle endurance.

Using the TPM3 mutant transgenic fish as a drug screening platform, we found L-carnitine specifically can restore the muscle endurance and swimming speed. Most of $\mathrm{L}$-carnitine is intracellular and is stored in liver, skeletal muscle, heart, and kidney [31]. L-Carnitine promotes long chain fatty acyl CoAs into the mitochondria for $\beta$-oxidation and nonoxidative glucose disposal $[32,33]$. Organic cation transporter-2 (OCTN2) enhances L-carnitine uptake inside cells. Carnitine acyltransferases are a family of enzymes that catalyze the reversible transfer of acyl groups between coenzyme A and L-carnitine and the conversion of acyl-CoA esters to acylcarnitine esters [34]. Neuroprotection was described in several animal models when administered with L-carnitine at super physiological concentrations. L-Carnitine was used to reduce lactate levels and elevate ATP levels upon administration [35]. A nemaline myopathy case with ACTA-1 gene mutation and carnitine deficiency was the first described with evidence of a disorder of mitochondrial fatty acid oxidation [36].

The pathogenesis of $\mathrm{CM}$ is diverse. Based on recent emerging evidence, however, five key pathophysiological areas have been proposed, including defects in sarcolemmal and intracellular membrane remodeling and excitation-contraction coupling; mitochondrial distribution and function; myofibrillar force generation; atrophy; and autophagy [7]. We hypothesized that high concentrations of L-carnitine can enhance long chain fatty acyl-CoA into 


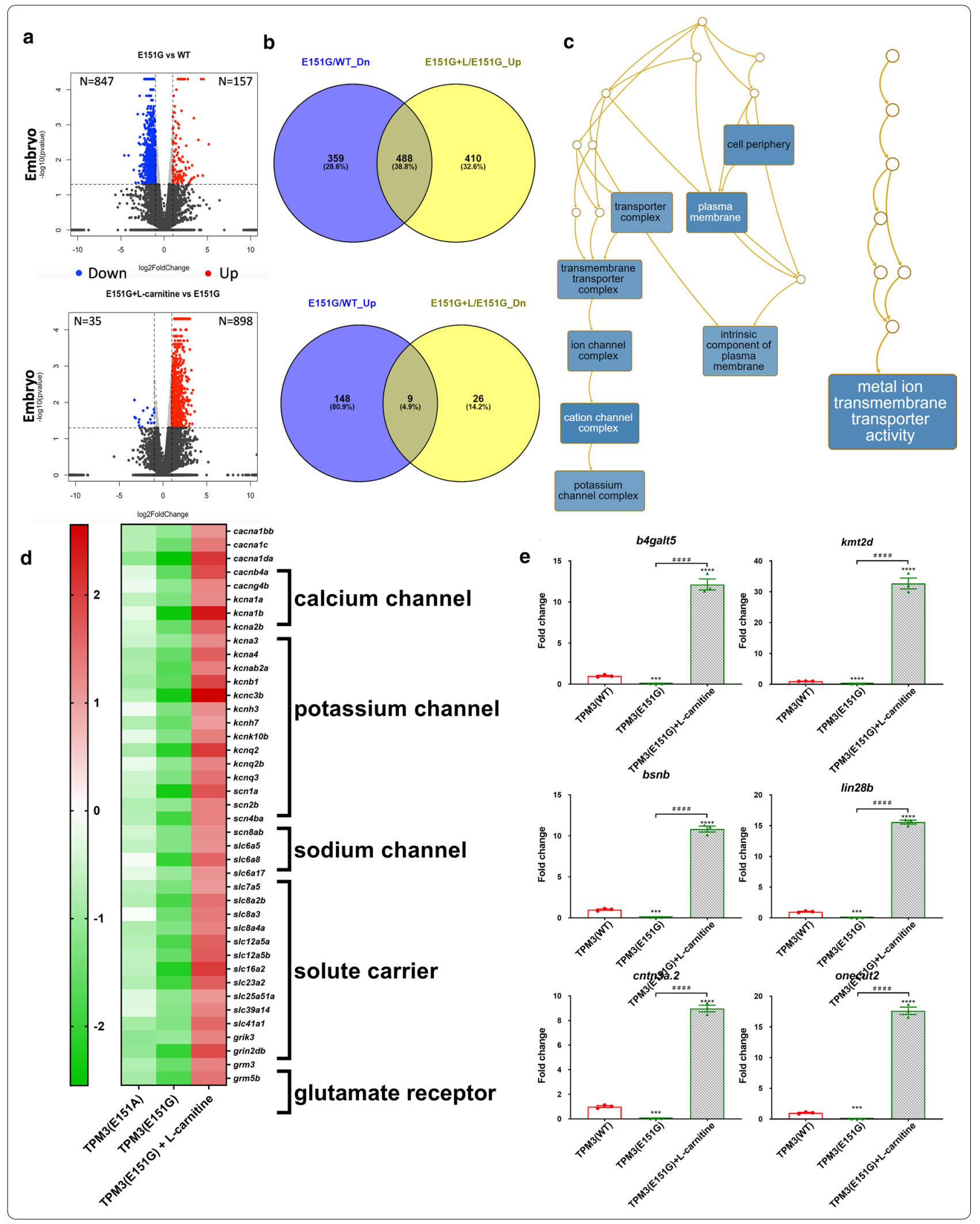




\section{(See figure on next page.)}

Fig. 7 Analysis and interpretation the embryos of TPM3(E151A) and TPM3(E151G) transgenic fish with ingenuity pathway analysis. a List canonical signaling pathways and metabolic pathways affected by the TPM3(E151A), TPM3(E151G) and L-carnitine treated TPM3(E151G) by IPA analysis presented as hierarchical clustering. $\mathbf{b}$ List the upstream molecules related to the changing molecules in the TPM3(E151A), TPM3(E151G) and L-carnitine treated TPM3(E151G), and predict whether they are activated or inhibited based on the research literature presented as hierarchical clustering. $\mathbf{c}$ List of various analytical diseases and biological functions dysregulated by TPM3(E151A), TPM3(E151G) and L-carnitine treated TPM3(E151G) presented as hierarchical clustering. $\mathbf{d}$ Fatty acid metabolism is downregulated in TPM3(E151G) (left) and upregulated by L-carnitine treatment in TPM3(E151G) (right). The genes differential expressed are shown in red or blue indicated up-regulated or downregulated respectively. The blue or orange color in the center indicated fatty acid metabolism are downregulated or upregulated respectively

the mitochondria for $\beta$-oxidation to generate ATP to rescue muscle weakness.

In our TPM3 transgenic zebrafish platform, we used larvae and adult fish for drug screening; some results from the larvae and adult fish platform seem unconcordant. We found that L-carnitine improve the swimming velocity of larval TPM3(E151G) zebrafish treated from $28 \mathrm{hpf}$ for seven days (Fig. 4a). The muscle endurance of adult TPM3(E151G) zebrafish was rescued to near normal levels in adult treated L-carnitine from $28 \mathrm{hpf}$ for one month (Fig. 4f), but L-carnitine could not rescue the muscle endurance of adult TPM3(E151G) zebrafish if treated at 3 months age (Additional file 4: Fig. S2A). It could be that adult fish muscle is well developed, the defect caused by TPM3(E151G) was unable to be rescued by L-carnitine if treated at 3 months of age. The myogenic regulatory factors, such as Myod, Myf5, and Myogenin, are expressed during the segmentation period [37, 38] at 1 day post fertilization during development [12], and decrease in adult stage. L-carnitine enhances long chain fatty acyl-CoAs into the mitochondria for $\beta$-oxidation to generate energy for skeletal muscle satellite (stem) cells to regenerate. The TPM3(E151G) larva zebrafish was rescued to near normal levels if treated L-carnitine from 28 hpf for seven days or one month. However, the stem cells are fewer with lower activity in adult of 3 month of age. Therefore, the muscle endurance cannot be improved in adult TPM3(E151G) zebrafish even if provided enough energy.

The sarcolemmal ion channels are crucial for muscle contraction involving excitation-contraction coupling machinery, sodium channelopathies are critical for myopathy, epilepsy disorders and complex encephalopathies [39]. Interestingly, sodium channel SCN4A mutations have been reported to be associated with severe fetal hypokinesia or classical CM [28]. In our study, we found genes involved in sodium channel: scn1a, scn2, scn $4 b a$ and $\operatorname{scn} 8 a b$ were upregulated by L-carnitine treatment (Fig. 6d). Calcium channel genes including calcium voltage-gated channel subunit alpha 1S (CACNA1S) [40], ryanodine receptor type 1 (RYR1) [41] and SH3 and cysteine rich domain 3 (STAC3) [42] and their mutations cause $\mathrm{CM}$ have been reported. In our study, we found calcium channel genes including: cacna $1 b b$, cacna1c, cacnalda, cacnb4a and cacng $4 b$ were downregulated in TPM3 mutation and their downregulation can be reverted by L-carnitine treatment (Fig. 6d). Mutations affects potassium channel has been reported to associate with myopathy in mouse [43]. In our study, we found potassium channel genes including: kcnala, kcna1b, kcna2b, kcna3, kcna4, kcnab2a, kcnb1, kcnc3b, kcnh3, $k c n h 7, k c n k 10 b, k c n q 2$, kcnq2b and kcnq3 were downregulated in TPM3(E151G) mutation and also can be reverted by L-carnitine treatment (Fig. 6d). The muscles of TPM3(E151G) larvae exhibits dysfunction of ion channels, L-carnitine treatment could reverse the gene expression of mainly sodium, potassium, and calcium channels, indicating that those ion channel genes may play key roles in pathophysiology of TPM3-related CM.

Although we found L-carnitine can improve the swimming velocity of larvae and the muscle endurance of TPM3(E151G) zebrafish if treated in the early stage for one month, treatment was not effective if started at three months of age. Clinically, human patients have often completed muscle development at the time of diagnosis, which is similar to adult fish. As with all CM, no FDAapproved treatment exists to date. Early implementation of NGS to enhance diagnostic capabilities of clinical suspected CM, especially overcome historic limitations of histopathologic and clinical overlap, must be developed so potential treatment can be administered as early as possible.

\section{Conclusions}

Our transgenic fish serve as translational model of TPM3 de novo mutation for a clinically diagnosed boy who suffers from CM. It is the first study using transgenic zebrafish to prove the pathogenicity of a TPM3(E151G) novel mutation of CM. Moreover, we demonstrate that L-carnitine ameliorates the muscle endurance 


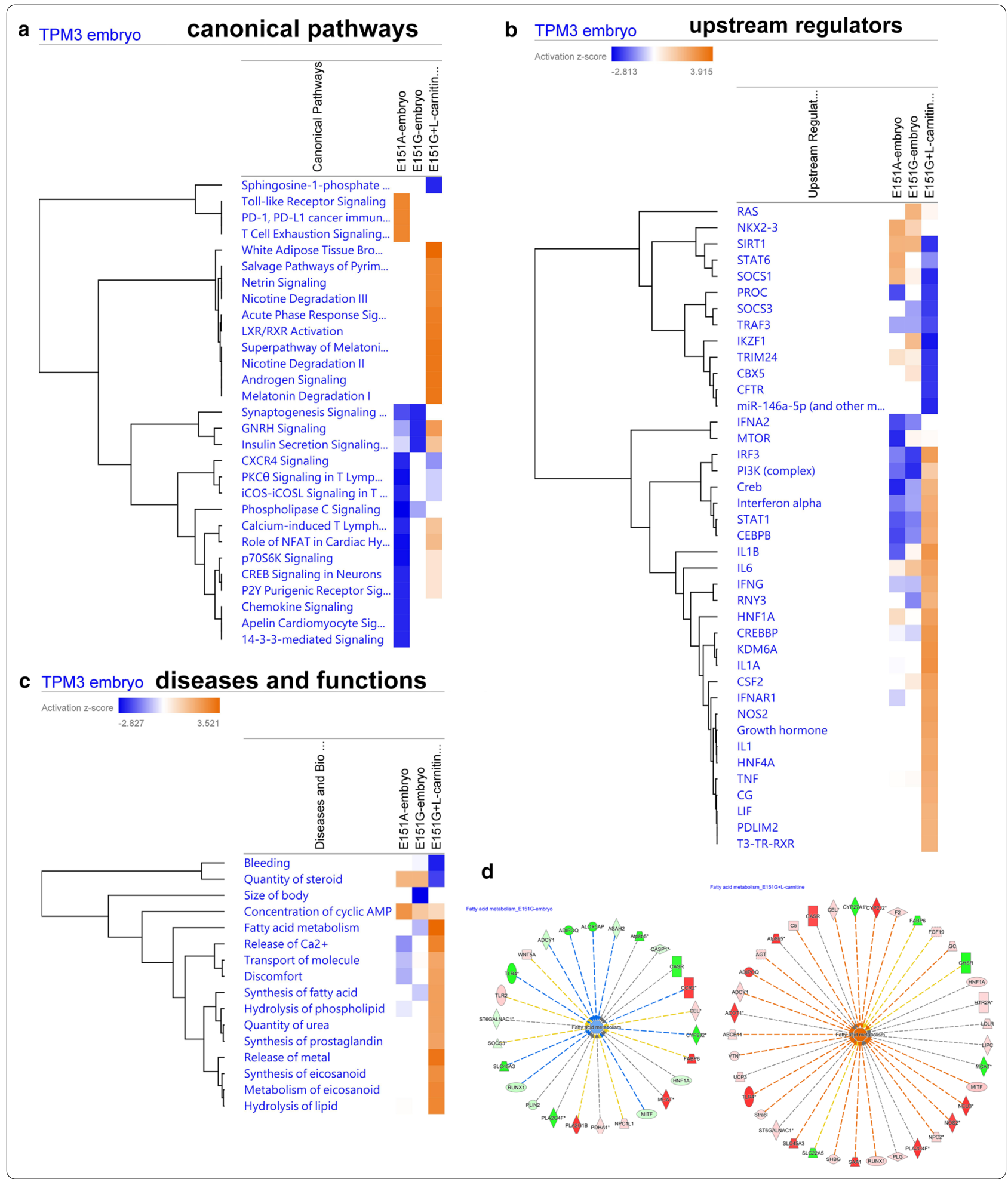

and pathophysiology of CM with TPM3(E151G) de novo mutation. This study highlights a role of potassium and calcium ion channels in TPM3-related CM and provides a potential mechanism of action of TPM3 pathophysiology. 


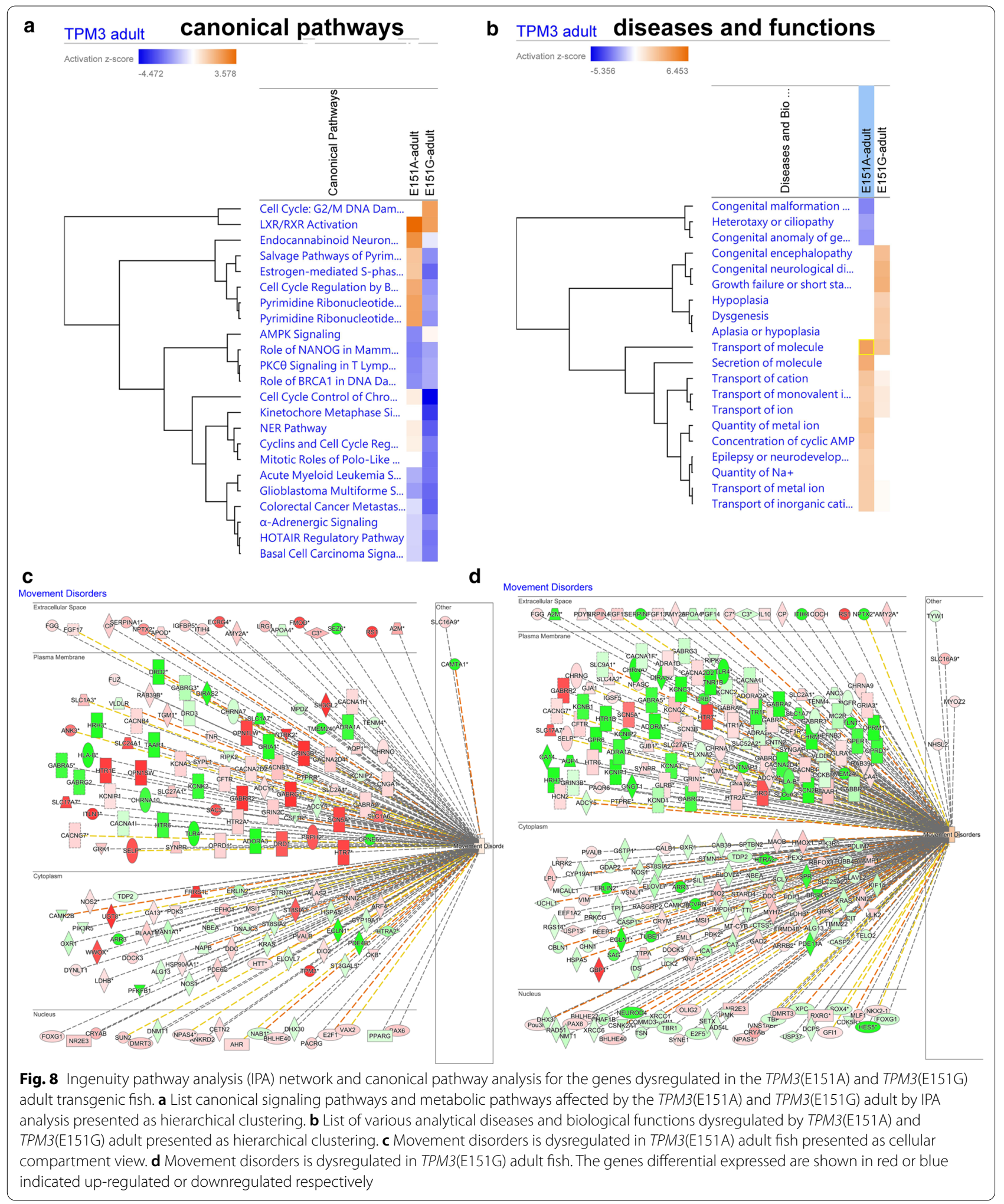




\section{Supplementary Information}

The online version contains supplementary material available at https://doi. org/10.1186/s12929-020-00707-1.

Additional file 1: Table S1. Primer information.

Additional file 2: Figure S1. The swimming velocity of larvae F1 TPM3 transgenic zebrafish at 3-6 dpf

Additional file 3: Movie S1. Swimming behavior of TPM3(E151A) and TPM3(E151G) compared with TPM3(WT) transgenic fish.

Additional file 4: Figure S2. L-carnitine treatment of F3 adult TPM3 transgenic zebrafish starting at 3 months of age.

Additional file 5: Figure S3. L-carnitine treatment increased the body weight and length of TPM3(E151G) larva.

Additional file 6: Figure S4. A heatmap showing the deep sequencing data for TPM3 transgenic zebrafish.

Additional file 7: Figure S5. The GRT-PCR validation of genes involved in anatomy structure development.

\section{Abbreviations}

TPM3: Tropomyosin 3; CM: Congenital myopathy; CFTD: Congenital fiber type disproportion; AAALAC: Assessment and Accreditation of Laboratory Animal Care International; NHRI: National Health Research Institutes; MLC2: Myosin light chain 2; $U_{\text {crit: }}$ Critical swimming speed; OCR: Oxygen consumption rate; FCCP: Carbonyl cyanide 4-(trifluoromethoxy)phenylhydrazone; PCA Principal component analysis; DMSO: Dimethyl sulfoxide; qRT-PCR: Quantitative real-time reverse transcription polymerase chain reaction; NADH-TR stain: Nicotinamide adenine dinucleotide dehydrogenase-tetrazolium reductase stain; HE stain: Hematoxylin and Eosin stain; CDNA: Complementary DNA; FPKM: Fragments per kilobase per million; KEGG: Kyoto encyclopedia of genes and genomes; TZCF: Taiwan Zebrafish Core Facility; ICOB: Institute of Cellular and Organismic Biology; dpf: Days post fertilization; MOHW: Ministry of Health and Welfare.

\section{Acknowledgements}

We thank Dr. Hsuan-Chin Ho for operating the X-ray, and the technicians in the National Museum of Marine Biology and Aquarium. In addition, we thank the Taiwan Zebrafish Core Facility (TZCF), which was supported by grant 104 2321-B-001-045 from MOST for the zebrafish maintenance. The support from "Taiwan Foundation For Rare Disorders" (2018, The 20th Rare Disease Master's Thesis Award) to Mr. Po-Jui Hsu is acknowledged.

\section{Authors' contributions}

PJH performed experiments, collected and analyzed the data. HDW design the experiment, contributes intellectual input, and edited the manuscript. YCT provided swimming tunnel, assisted muscle endurance experiments, and analysis of results. SWP performed the histopathological staining for $A B(W T)$ and L-carnitine treatment. BPS performed the qRT-PCR for type I and type II muscle. YJJ and CHY supervision, designed the study, writing and editing the manuscript, funding acquisition. All authors read and approved the final manuscript.

\section{Funding}

This study was supported by a grant from the Taiwan Ministry of Science and Technology (MOST 106-2314-B-037-100-MY3) to Dr. Yuh-Jyh Jong and financial support from the National Health Research Institutes (MG-109-PP-06) and Taiwan's Ministry of Health and Welfare (MOHW), Advance Medical Plan (108-0324-01-19-07), Taiwan Ministry of Science and Technology (MOST 1082320-B-400 -005-MY3) to Dr. Chiou-Hwa Yuh.

\section{Availability of data and materials}

The NGS data have been submitted to the NCBI Gene Expression Omnibus (GEO) under accession code GSE149261.

\section{Ethics approval and consent to participate}

Not applicable.

\section{Consent for publication}

Not applicable.

\section{Competing interests}

The authors declare that they have no competing interests.

\begin{abstract}
Author details
${ }^{1}$ Institute of Molecular and Genomic Medicine, National Health Research Institutes, Zhunan, Miaoli, Taiwan. ${ }^{2}$ Institute of Biotechnology, National Tsing Hua University, Hsinchu, Taiwan. ${ }^{3}$ Department of Laboratory Medicine, Mackay Memorial Hospital, Taipei, Taiwan. ${ }^{4}$ Marine Research Station, Institute of Cellular and Organismic Biology, Academia Sinica, I-Lan County, Taiwan. ${ }^{5}$ Department of Biological Science and Technology, National Chiao Tung University, Hsinchu, Taiwan. ${ }^{6}$ Graduate Institute of Clinical Medicine, College of Medicine, Kaohsiung Medical University, Kaohsiung, Taiwan. ${ }^{7}$ Departments of Pediatrics and Laboratory Medicine, Kaohsiung Medical University, Kaohsiung, Taiwan. ${ }^{8}$ Translational Research Center of Neuromuscular Diseases, Kaohsiung Medical University, Kaohsiung, Taiwan. ${ }^{9}$ Institute of Bioinformatics and Structural Biology, National Tsing Hua University, Hsinchu, Taiwan. ${ }^{10}$ Ph.D. Program in Environmental and Occupational Medicine, Kaohsiung Medical University, Kaohsiung, Taiwan.
\end{abstract}

Received: 10 July 2020 Accepted: 30 December 2020

Published online: 12 January 2021

\section{References}

1. North KN, Wang $\mathrm{CH}$, Clarke N, Jungbluth H, Vainzof M, Dowling JJ, et al. Approach to the diagnosis of congenital myopathies. Neuromuscul Disord. 2014;24(2):97-116.

2. Winter JM, Joureau B, Lee EJ, Kiss B, Yuen M, Gupta VA, et al. Mutationspecific effects on thin filament length in thin filament myopathy. Ann Neurol. 2016;79(6):959-69.

3. Marttila M, Lehtokari VL, Marston S, Nyman TA, Barnerias C, Beggs AH, et al. Mutation update and genotype-phenotype correlations of novel and previously described mutations in TPM2 and TPM3 causing congenital myopathies. Hum Mutat. 2014;35(7):779-90.

4. Yuen M, Cooper ST, Marston SB, Nowak KJ, McNamara E, Mokbel N, et al. Muscle weakness in TPM3-myopathy is due to reduced Ca2+-sensitivity and impaired acto-myosin cross-bridge cycling in slow fibres. Hum Mol Genet. 2015;24(22):6278-92.

5. Moraczewska J, Robaszkiewicz K, Sliwinska M, Czajkowska M, Ly T, Kostyukova A, et al. Congenital myopathy-related mutations in tropomyosin disrupt regulatory function through altered actin affinity and tropomodulin binding. FEBS J. 2019;286(10):1877-93.

6. Benarroch L, Bonne G, Rivier F, Hamroun D. The 2020 version of the gene table of neuromuscular disorders (nuclear genome). Neuromuscul Disord. 2019;29(12):980-1018.

7. Ravenscroft G, Laing NG, Bonnemann CG. Pathophysiological concepts in the congenital myopathies: blurring the boundaries, sharpening the focus. Brain. 2015;138(Pt 2):246-68.

8. Zhao M, Smith L, Volpatti J, Fabian L, Dowling JJ. Insights into wild-type dynamin 2 and the consequences of DNM2 mutations from transgenic zebrafish. Hum Mol Genet. 2019;28(24):4186-96.

9. Ale MT, Mikkelsen JD, Meyer AS. Important determinants for fucoidan bioactivity: a critical review of structure-function relations and extraction methods for fucose-containing sulfated polysaccharides from brown seaweeds. Mar Drugs. 2011;9(10):2106-30.

10. Jang J, Park J, Chang H, Lim K. I-Carnitine supplement reduces skeletal muscle atrophy induced by prolonged hindlimb suspension in rats. Appl Physiol Nutr Metab. 2016;41(12):1240-7.

11. Gimenes AC, Bravo DM, Napolis LM, Mello MT, Oliveira AS, Neder JA, et al. Effect of L-carnitine on exercise performance in patients with mitochondrial myopathy. Braz J Med Biol Res. 2015;48(4):354-62.

12. Ochi $H$, Westerfield $M$. Signaling networks that regulate muscle development: lessons from zebrafish. Dev Growth Differ. 2007;49(1):1-11.

13. Kimmel CB, Kane DA, Walker C, Warga RM, Rothman MB. A mutation that changes cell movement and cell fate in the zebrafish embryo. Nature. 1989;337(6205):358-62. 
14. Messineo AM, Gineste C, Sztal TE, McNamara EL, Vilmen C, Ogier AC, et al. L-tyrosine supplementation does not ameliorate skeletal muscle dysfunction in zebrafish and mouse models of dominant skeletal muscle alpha-actin nemaline myopathy. Sci Rep. 2018;8(1):11490.

15. Tian X, Liang WC, Feng Y, Wang J, Zhang VW, Chou CH, et al. Expanding genotype/phenotype of neuromuscular diseases by comprehensive target capture/NGS. Neurol Genet. 2015;1(2):e14.

16. You MS, Jiang YJ, Yuh CH, Wang CM, Tang CH, Chuang YJ, et al. A sketch of the Taiwan zebrafish core facility. Zebrafish. 2016;13(Suppl 1):S24-9.

17. Lu JW, Yang WY, Tsai SM, Lin YM, Chang PH, Chen JR, et al. Liver-specific expressions of $\mathrm{HBx}$ and src in the $\mathrm{p} 53$ mutant trigger hepatocarcinogenesis in zebrafish. PLoS ONE. 2013;8(10):e76951.

18. Chou YT, Jiang JK, Yang MH, Lu JW, Lin HK, Wang HD, et al. Identification of a noncanonical function for ribose-5-phosphate isomerase A promotes colorectal cancer formation by stabilizing and activating betacatenin via a novel C-terminal domain. PLoS Biol. 2018;16(1):e2003714.

19. Shao YT, Chuang SY, Chang HY, Tseng YC, Shao KT. Largescale mullet (Planiliza macrolepis) can recover from thermal pollution-induced malformations. PLoS ONE. 2018;13(11):e0208005.

20. Liu Y, Ma P, Cassidy PA, Carmer R, Zhang G, Venkatraman P, et al. Statistical analysis of zebrafish locomotor behaviour by generalized linear mixed models. Sci Rep. 2017;7(1):2937.

21. Gao Y, Chan RH, Chow TW, Zhang L, Bonilla S, Pang CP, et al. A highthroughput zebrafish screening method for visual mutants by lightinduced locomotor response. IEEE/ACM Trans Comput Biol Bioinform. 2014;11(4):693-701.

22. Brett JR. The respiratory metabolism and swimming performance of Young Sockeye Salmon. J Fish Res Board Can. 1964;21(5):1183-226.

23. Sztal TE, McKaige EA, Williams C, Oorschot V, Ramm G, Bryson-Richardson RJ. Testing of therapies in a novel nebulin nemaline myopathy model demonstrate a lack of efficacy. Acta Neuropathol Commun. 2018:6(1):40.

24. Boyd PJ, Tu WY, Shorrock HK, Groen EJN, Carter RN, Powis RA, et al. Bioenergetic status modulates motor neuron vulnerability and pathogenesis in a zebrafish model of spinal muscular atrophy. PLoS Genet. 2017;13(4):e1006744

25. Sztal TE, Currie PD, Bryson-Richardson RJ. Analysis of RNA expression in adult zebrafish skeletal muscle. Methods Mol Biol. 2017;1668:27-35

26. Liao Y, Wang J, Jaehnig EJ, Shi Z, Zhang B. WebGestalt 2019: gene set analysis toolkit with revamped UIs and APIs. Nucleic Acids Res. 2019;47(W1):W199-205.

27. Tajsharghi H, Oldfors A. Myosinopathies: pathology and mechanisms. Acta Neuropathol. 2013;125(1):3-18.

28. Zaharieva IT, Thor MG, Oates EC, van Karnebeek C, Hendson G, Blom E, et al. Loss-of-function mutations in SCN4A cause severe foetal hypokinesia or "classical" congenital myopathy. Brain. 2016;139(Pt 3):674-91.

29. Levin ED, Cerutti DT. Behavioral neuroscience of zebrafish. In: Buccafusco $\mathrm{JJ}$, editor. Methods of behavior analysis in neuroscience. Boca Raton: CRC Press; 2009

30. Prakash J, Guruprasad CM. T-maze alternation for zebrafish behavioural studies. World J Pharm Pharm Sci. 2017;6(11):361-6.
31. Jones $L L, M c D o n a l d ~ D A$, Borum PR. Acylcarnitines: role in brain. Prog Lipid Res. 2010;49(1):61-75.

32. Alves E, Binienda Z, Carvalho F, Alves CJ, Fernandes E, de Lourdes BM, et al. Acetyl-L-carnitine provides effective in vivo neuroprotection over 3,4-methylenedioximethamphetamine-induced mitochondrial neurotoxicity in the adolescent rat brain. Neuroscience. 2009;158(2):514-23.

33. Cahova M, Chrastina P, Hansikova H, Drahota Z, Trnovska J, Skop V, et al. Carnitine supplementation alleviates lipid metabolism derangements and protects against oxidative stress in non-obese hereditary hypertriglyceridemic rats. Appl Physiol Nutr Metab. 2015;40(3):280-91.

34. Adeva-Andany MM, Calvo-Castro I, Fernandez-Fernandez C, DonapetryGarcia C, Pedre-Pineiro AM. Significance of I-carnitine for human health. IUBMB Life. 2017;69(8):578-94.

35. Zanelli SA, Solenski NJ, Rosenthal RE, Fiskum G. Mechanisms of ischemic neuroprotection by acetyl-L-carnitine. Ann NY Acad Sci. 2005;1053:153-61.

36. Laing NG, Dye DE, Wallgren-Pettersson C, Richard G, Monnier N, Lillis S, et al. Mutations and polymorphisms of the skeletal muscle alpha-actin gene (ACTA1). Hum Mutat. 2009;30(9):1267-77.

37. Weinberg ES, Allende ML, Kelly CS, Abdelhamid A, Murakami T, Andermann P, et al. Developmental regulation of zebrafish MyoD in wild-type, no tail and spadetail embryos. Development. 1996;122(1):271-80.

38. Coutelle O, Blagden CS, Hampson R, Halai C, Rigby PW, Hughes SM. Hedgehog signalling is required for maintenance of myf5 and myoD expression and timely terminal differentiation in zebrafish adaxial myogenesis. Dev Biol. 2001;236(1):136-50.

39. Matthews E, Balestrini S, Sisodiya SM, Hanna MG. Muscle and brain sodium channelopathies: genetic causes, clinical phenotypes, and management approaches. Lancet Child Adolesc Health. 2020;4(7):536-47.

40. Schartner V, Romero NB, Donkervoort S, Treves S, Munot P, Pierson TM, et al. Dihydropyridine receptor (DHPR, CACNA1S) congenital myopathy. Acta Neuropathol. 2017;133(4):517-33.

41. Lawal TA, Todd JJ, Meilleur KG. Ryanodine receptor 1-related myopathies: diagnostic and therapeutic approaches. Neurotherapeutics. 2018;15(4):885-99.

42. Zaharieva IT, Sarkozy A, Munot P, Manzur A, O'Grady G, Rendu J, et al. STAC3 variants cause a congenital myopathy with distinctive dysmorphic features and malignant hyperthermia susceptibility. Hum Mutat. 2018;39(12):1980-94.

43. Hanson MG, Wilde JJ, Moreno RL, Minic AD, Niswander L. Potassium dependent rescue of a myopathy with core-like structures in mouse. Elife. 2015. https://doi.org/10.7554/eLife.02923.

\section{Publisher's Note}

Springer Nature remains neutral with regard to jurisdictional claims in published maps and institutional affiliations.

Ready to submit your research? Choose BMC and benefit from

- fast, convenient online submission

- thorough peer review by experienced researchers in your field

- rapid publication on acceptance

- support for research data, including large and complex data types

- gold Open Access which fosters wider collaboration and increased citations

- maximum visibility for your research: over $100 \mathrm{M}$ website views per year

At BMC, research is always in progress.

Learn more biomedcentral.com/submissions 